\title{
THE ACS SURVEY OF GALACTIC GLOBULAR CLUSTERS. VIII. EFFECTS OF ENVIRONMENT ON GLOBULAR CLUSTER GLOBAL MASS FUNCTIONS
}

\author{
Nathaniel E. Q. Paust ${ }^{1}$, I. Neill Reid ${ }^{2}$, Giampaolo Piotto ${ }^{3}$, Antonio Aparicio ${ }^{4,5}$, Jay Anderson ${ }^{2}$, Ata Sarajedini ${ }^{6}$, \\ Luigi R. Bedin ${ }^{2}$, Brian Chaboyer ${ }^{7}$, Aaron DotTer $^{8}$, Maren Hempel $^{6}$, STeven Majewski $^{9}$, A. Marín-Franch $^{5}$, \\ Antonino Milone ${ }^{3}$, Alfred Rosenberg $^{5}$, and Michael Siegel $^{10,11}$ \\ ${ }^{1}$ Whitman College, 345 Boyer Avenue, Walla Walla, WA 99362, USA; paustne@ whitman.edu \\ ${ }^{2}$ Space Telescope Science Institute, 3700 San Martin Drive, Baltimore, MD 21218, USA; inr@ stsci.edu, anderson@stsci.edu, bedin@stsci.edu \\ ${ }_{3}^{3}$ Dipartimento di Astronomia, University di Padova, vicolo dell'Osservatorio 5, I-35122 Padova, Italy; piotto@pd.astro.it, milone@pd.astro.it \\ ${ }^{4}$ Department of Astrophysics, University of La Laguna, Vía Láctea s/n, E-38200 La Laguna, Spain \\ ${ }^{5}$ Instituto de Astrofísica de Canarias, Vía Láctea s/n, E-38200 La Laguna, Spain; antapaj@iac.es, amarin@iac.es, alf@iac.es \\ ${ }^{6}$ Department of Astronomy, University of Florida, 211 Bryant Space Science Center, Gainesville, FL 32611, USA; ata@ astro.ufl.edu, hempel@ astro.ufl.edu \\ ${ }^{7}$ Department of Physics and Astronomy, Dartmouth College, 6127 Wilder Laboratory, Hanover, NH 03755, USA; chaboyer@ heather.dartmouth.edu \\ ${ }^{8}$ Department of Physics and Astronomy, University of Victoria, Elliott Building, 3800 Finnerty Road, Victoria, BC V8P 5C2, Canada; dotter@uvic.ca \\ ${ }^{9}$ Department of Astronomy, University of Virginia, P.O. Box 400325, Charlottesville, VA 22904-4325, USA; srm4n@ virginia.edu \\ ${ }^{10}$ University of Texas, McDonald Observatory, 1 University Station, C1402, Austin, TX 78712, USA; siegel@ astro.psu.edu \\ Received 2009 February 2; accepted 2009 November 18; published 2010 January 8
}

\begin{abstract}
We have used observations obtained as part of the Hubble Space Telescope/ACS Survey of Galactic Globular Clusters to construct global present-day mass functions for 17 globular clusters utilizing multi-mass King models to extrapolate from our observations to the global cluster behavior. The global present-day mass functions for these clusters are well matched by power laws from the turnoff, $\approx 0.8 M_{\odot}$, to $0.2-0.3 M_{\odot}$ on the lower main sequence. The slopes of those power-law fits, $\alpha$, have been correlated with an extensive set of intrinsic and extrinsic cluster properties to investigate which parameters may influence the form of the present-day mass function. We do not confirm previous suggestions of correlations between $\alpha$ and either metallicity or Galactic location. However, we do find a strong statistical correlation with the related parameters central surface brightness, $\mu_{V}$, and inferred central density, $\rho_{0}$. The correlation is such that clusters with denser cores (stronger binding energy) tend to have steeper mass functions (a higher proportion of low-mass stars), suggesting that dynamical evolution due to external interactions may have played a key role in determining $\alpha$. Thus, the present-day mass function may owe more to nurture than to nature. Detailed modeling of external dynamical effects is therefore a requisite for determining the initial mass function for Galactic globular clusters.
\end{abstract}

Key words: globular clusters: individual (NGC 1261, NGC 1851, NGC 5466, NGC 5927, NGC 6093, NGC 6341, NGC 6362, NGC 7099)

\section{INTRODUCTION}

Globular clusters are among the oldest structures in the universe that are accessible to direct observational investigation. With metallicities ranging from $[\mathrm{Fe} / \mathrm{H}] \sim-2.4$ to -0.5 dex or higher and ages from $\approx 11$ to $13 \mathrm{Gyr}$, these systems originated in the earliest stages of the formation of the Milky Way. Until recently, globulars were regarded as simple stellar populations, products of a single-epoch, uniform-metallicity star-forming burst. However, high-precision photometry, primarily with the Hubble Space Telescope (HST), has led to the identification of multiple components on the main sequence and/or the subgiant branch in a number of massive globulars, including $\omega$ Cen (Bedin et al. 2004), NGC 1851 (Milone et al. 2008), and NGC 2808 (Piotto et al. 2007). Several systems, such as $\omega$ Cen (Villanova et al. 2007) and M54 (Siegel et al. 2007), may, in fact, be remnant nuclei of dwarf galaxies disrupted through gravitational interactions with the Milky Way. At the same time, detailed spectroscopic analyses of other clusters have revealed abundance anomalies that have been interpreted as symptomatic of more complex formation histories (Gratton et al. 2004).

Even with these complications, globular clusters still offer crucial insight into the conditions and mechanisms present in

\footnotetext{
${ }^{11}$ Current address: Pennsylvania State University, 525 Davey Lab, State College, PA 16801, USA.
}

the nascent Milky Way. A key question centers on the star formation process, specifically, the number distribution of stars as a function of mass, the initial stellar mass function (IMF), and how that function may have changed over the Milky Way's history. Over the last decade, detailed investigations of nearby stars and open clusters have shown that the IMF in the metalrich Galactic disk is well matched by a near-Salpeter slope power law $(\alpha=2.35)$ at high masses, flattening to $\alpha \approx 1$ at $\approx 1 M_{\odot}$, and turning over at $\approx 0.15-0.1 M_{\odot}$, near the brown dwarf regime. Most of the mass lies in sub-solar-mass stars, with the median value near $\sim 0.3 M_{\odot}$ (Reid \& Gizis 1997; Reid et al. 2002; Kroupa 2002; Andersen et al. 2008). Theoretical expectations are that the characteristic mass should increase at lower metallicities, as cooling becomes more difficult and the Jeans mass increases (Larson 1998; Bromm \& Loeb 2003). Indeed, recent studies suggest that the first stars, forming from unenriched $\mathrm{H} / \mathrm{He}$ gas, had typical masses exceeding $100 M_{\odot}$ (Bromm et al. 2002). Globular clusters provide an obvious means of probing this issue and investigating changes in the stellar IMF with metallicity as the clusters formed quite early.

Determining the global present-day mass function of a globular cluster is a complicated task, however, that requires not only deep photometry in extremely crowded fields and careful attention to completeness, but also dynamical modeling of the underlying stellar distribution. As discussed originally 
by King (1958), internal relaxation results in mass segregation, with higher mass stars concentrated toward the cluster core; subsequent external dynamical encounters lead to preferential stripping of lower mass stars, with consequent effect on the global cluster mass function. Dynamical modeling can be used to correct for internal mass segregation, permitting the derivation of the present-day global mass function (King et al. 1995; Anderson 1997), but reconstructing the past history of tidal stripping and the IMF is extremely difficult, even given full knowledge of the cluster's Galactic orbit. Nonetheless, past studies have examined correlations between the shape of the present-day cluster mass function and other intrinsic and extrinsic parameters, including metallicity, concentration, and location in the Galaxy (Capaccioli et al. 1993; Djorgovski et al. 1993; Piotto \& Zoccali 1999).

The $H S T$ has proven an extremely effective tool for investigating the properties of globular clusters. With that in mind, we initiated a Cycle 14 HST Treasury program, using the Advanced Camera for Surveys Wide Field Channel (ACS/WFC), designed to provide deep, homogeneous data for a representative subsample of the cluster population (Sarajedini et al. 2007). The ACS Galactic Globular Cluster (ACS GGC) Survey includes 65 clusters, with the prime selection criteria distance $(d<20 \mathrm{kpc}$, or $\left.(m-M)_{0}<16.5\right)$ and low reddening $(E(B-V) \leqslant 0.35)$. The observations cover the central regions of each cluster, and extend to $V \sim 26.5$ and $I \sim 25$, corresponding to masses $\approx 0.25 M_{\odot}$ in most clusters.

This paper presents global present-day mass functions for 17 globulars from the ACS GGC chosen at random from the non-core-collapsed clusters observed in the project. Clusters with observed multiple stellar populations were also excluded. Over the past 18 years, the HST has invested hundreds of orbits in observations of numerous GGCs, although only a subset of those observations are of sufficient depth to allow accurate determination of the luminosity function and mass function on the lower main sequence. Section 2 summarizes the main results from those previous $H S T$ investigations. In Section 3, we describe the observations along with the photometry method used and the determination of completeness. Section 4 describes the methodology used to extrapolate from our observations of the cluster cores to the global cluster behaviors, and Section 5 applies those methods to derive global present-day mass functions for each cluster in the present sample. Section 6 examines the correlations between the mass function slope and a wide range of other cluster parameters, Section 7 discusses the implications of those results, and Section 8 summarizes our conclusions.

\section{HST AND GLOBULAR CLUSTER LUMINOSITY FUNCTIONS}

\subsection{Previous Observations with HST}

The unparalleled angular resolution at optical wavelengths offered by HST was recognized from the outset as a key advantage in probing the intrinsic parameters of globular clusters (e.g., Bahcall \& Schneider 1988). Here, we review the results from previous investigations, with a particular focus on analyses of the stellar mass function, to place the ACS GGC analysis in the appropriate context.

The spherical aberration present in Hubble's primary mirror severely limited the scope of the initial observing programs, which focused, perforce, on UV observations of hot, bright stars in cluster cores. Thus, de Marchi \& Paresce (1994) used the Faint Object Camera (FOC) to identify blue stragglers within the central 2 arcsec of M15, while Guhathakurta et al. (1994) employed the Wide Field/Planetary Camera (WF/PC) to survey blue stragglers and variable stars (primarily interacting binaries) in M3. Other clusters studied include 47 Tuc (Guhathakurta et al. 1992; WF/PC), NGC 6397 (de Marchi \& Paresce 1994; Burgarella et al. 1994; both FOC), and NGC 6624 (Sosin \& Cool 1995; FOC).

Hubble only realized its full potential for globular cluster science with the installation of the Wide Field Planetary Camera 2 (WFPC2) during the first Servicing Mission in 1993 December. At that time, a key question centered on the numbers of low-mass subdwarfs and the shape of the mass function below $\approx 0.5 M_{\odot}$. Deep ground-based imaging of several clusters, including NGC 6397 (Fahlman et al. 1989), M30 (Richer et al. 1988), and M71 (Richer \& Fahlman 1988), suggested that the luminosity function was flat at $M_{V}>12$, implying a steeply rising mass function; characterized as a power law, $\Psi(M) \propto M^{-\alpha}$, the inferred index was close to the Salpeter value, $\alpha \sim 2.3$ (Richer et al. 1991; although see, e.g., Piotto et al. 1990 for a contrary analysis of ground-based data). In contrast, WFPC2 observations of NGC 6397 (Paresce et al. 1995b; Cool et al. 1996) revealed decreasing numbers of stars at low luminosities. The derived mass function was significantly flatter with $\alpha \sim 1$ for masses $M<0.3 M_{\odot}$, similar to $\Psi(M)$ for local disk stars. Subsequent analyses of $\omega$ Cen (Elson et al. 1995), 47 Tuc (de Marchi \& Paresce 1995a), and M15 (de Marchi \& Paresce 1995b) confirmed these results, demonstrating that the Richer et al. (1991) conclusions stemmed from overcorrecting for image confusion in the ground-based data.

The high angular resolution and stable point-spread function (PSF) offered by HST played an important role in probing mass segregation in globular clusters. In the dense cluster environment, two-body interactions equilibrate the kinetic energy of the stars, giving low-mass stars higher velocities and high-mass stars lower velocities. As a consequence, blue stragglers and horizontal branch stars are concentrated in the cluster cores, while low-mass M subdwarfs dominate at larger radii. Originally detected by Sandage (1954) and Tayler (1954) in M3 and M92, respectively, the theoretical underpinnings of this dynamical process were developed by Oort \& van Herk (1959). Da Costa (1982) provided the first extensive discussion of the phenomenon in his analysis of ground-based observations of 47 Tuc. Early HST imaging programs confirmed the results for 47 Tuc (Paresce et al. 1995a) and verified the presence of extensive mass segregation in other clusters (e.g., NGC 6397; King et al. 1995).

Over the subsequent 13 years, $H S T$ has been used to probe the subdwarf luminosity and mass function in the 17 clusters listed in Table 1. For this compilation, we only consider observations made after Servicing Mission 1 (SM1), and we have not included analyses that extend less than $\sim 2$ mag below the main-sequence turnoff (MSTO). The observations generally reach limits of $M_{I} \sim 11$, corresponding to mass limits between 0.3 and $0.35 M_{\odot}$. In one case, NGC 2298, the ACS data are taken from the current survey. The deepest data sets, for NGC 6397 and $\mathrm{M} 4$, the two nearest globular clusters, are derived from recent $100+$ orbit campaigns with WFPC 2 and ACS that were designed to probe the cluster white dwarf sequences. The NGC 6397 data extend well beyond the hydrogen-burning limit and take advantage of proper motions, derived from multiepoch observations, to determine improved membership criteria for the MS cluster members. This technique, pioneered by King et al. (1998), is particularly important in separating low 
Table 1

Globular Cluster Luminosity Functions from HST Data

\begin{tabular}{lcccccccc}
\hline \hline NGC & Other & {$[\mathrm{Fe} / \mathrm{H}]$} & $(m-M)_{0}$ & $A_{I}$ & Refs. & Instr. & Lim. Mag. & Refs. \\
\hline 104 & 47 Tuc & -0.66 & 13.42 & 0.04 & A & WFPC2 & $I \sim 24$ & 3 \\
288 & & -1.05 & 14.95 & 0.06 & B & WFPC2, NIC & $V \sim 25, H \sim 24$ & 18,22 \\
2298 & & -1.85 & 15.15 & 0.24 & G & ACS & $I \sim 25$ & 27 \\
5139 & W Cen & -1.60 & 13.72 & 0.20 & C & WFPC2 & $I \sim 26$ & 2,13 \\
5272 & M3 & -1.34 & 15.07 & 0.01 & C & WFPC2 & $I \sim 25$ & 10 \\
6121 & M4 & -1.19 & 11.39 & 0.63 & C & WFPC2, NIC, ACS & $I \sim 27.5, H \sim 24$ & $12,20,21$ \\
6254 & M10 & -1.41 & 13.38 & 0.39 & D & WFPC2 & $I \sim 25$ & 17 \\
6341 & M92 & -2.15 & 14.65 & 0.035 & B & WFPC2 & $I \sim 25$ & 7,16 \\
6352 & & -0.5 & 13.6 & 0.43 & E & WFPC2 & $I \sim 24.5$ & 24 \\
6397 & & -2.03 & 11.96 & 0.32 & A & WFPC2, NIC, ACS & $I \sim 29, H \sim 23$ & $1,5,7,11$ \\
& & & & & & WFPC2 & $I \sim 25.5$ & 15,28 \\
6496 & & -0.5 & 14.8 & 0.43 & E & WFPC2, ACS & $I \sim 25$ & 24 \\
6656 & M22 & -1.53 & 12.65 & 0.66 & C & WFP, & 9,23 \\
6752 & & -1.43 & 13.10 & 0.07 & A & WFPC2 & $I \sim 25$ & 8 \\
6809 & M55 & -1.61 & 13.62 & 0.13 & C & WFPC2 & $I \sim 25$ & 17 \\
7078 & M15 & -2.15 & 15.38 & 0.15 & B & WFPC2, NIC & $I \sim 25, H \sim 23$ & $4,7,25$ \\
7099 & M30 & -1.88 & 14.78 & 0.06 & B & WFPC2 & $I \sim 25$ & 7 \\
& Pal 5 & -1.41 & 16.83 & 0.06 & F & WFPC2 & $I \sim 25$ & 19 \\
\hline
\end{tabular}

Notes. All of the clusters listed above were observed by HST after SM1. WFPC2 observations also exist for NGC 6362 and 6394 (Piotto et al. 1999), but those observations are not included since they are limited to $V \sim 22$, only 1-2 mag below the MSTO. References for distances, metallicities, and reddening: (A) Gratton et al. 2003; (B) Carretta et al. 2000; (C) Sollima et al. 2006; (D) Pollard et al. 2005; (E) Pulone et al. 2003; (F) Grillmair \& Smith 2001; (G) de Marchi \& Pulone 2007. References for HST imaging: (1) Paresce et al. 1995b; (2) Elson et al. 1995; (3) de Marchi \& Paresce 1995a; (4) de Marchi \& Paresce 1995b; (5) Cool et al. 1996; (6) Santiago et al. 1996; (7) Piotto et al. 1997; (8) Ferraro et al. 1997; (9) de Marchi \& Paresce 1997; (10) Marconi et al. 1998; (11) King et al. 1998; (12) Pulone et al. 1999; (13) de Marchi 1999; (14) Piotto \& Zoccali 1999; (15) de Marchi et al. 2000; (16) Andreuzzi et al. 2000; (17) Paresce \& de Marchi 2000; (18) Pasquali et al. 2000; (19) Grillmair \& Smith 2001; (20) Bedin et al. 2001; (21) Richer et al. 2002; (22) Bellazzini et al. 2002; (23) Albrow et al. 2002; (24) Pulone et al. 2003; (25) Pasquali et al. 2004; (26) Sollima et al. 2007; (27) de Marchi \& Pulone 2007; (28) Richer et al. 2008.

luminosity cluster members from background field stars in these low galactic latitude clusters.

All the clusters have observations in the F814W passband, with the observations transformed in the original papers to the Cousins $I$ band, usually using the relations given by Holtzman et al. (1995). We have combined the published results with the distance and reddening limits cited in Table 1 to derive luminosity functions. We have taken the star counts directly from the published paper with the deepest results, correcting for completeness according to the scheme given in that paper. The resulting luminosity functions (excluding NGC 2298, which will be discussed further in a later paper in this series) are shown in Figure 1. Each set of observations provides only partial areal coverage of the cluster. The resultant luminosity functions, plotted in Figure 1, show a diverse range of properties. Most peak near $M_{I} \sim 8$, but some increase monotonically through the full magnitude range. In a few cases (NGC 288, M22, Pal 5, M92), the published analyses provide spatially resolved star counts within the cluster. We show those results separately in Figure 1, using crosses to mark data taken at larger radii from the cluster center.

\subsection{Characterizing the Mass Function}

It is important to recognize that the luminosity functions plotted in Figure 1 are drawn from inhomogeneous sources and have not been corrected for dynamical effects. Consequently, they represent local, present-day luminosity functions for the regions within the cluster covered by the HST observations. Nonetheless, these data provide a means of making a preliminary investigation of how one might best characterize cluster luminosity functions.
To that end, we have combined the I-band luminosity function data, $\Phi\left(M_{I}\right)$, with appropriate mass-luminosity relations derived from the theoretical models by Dotter et al. (2007). As discussed by Paust et al. (2009), those models are in good agreement with the recent set computed by Pietrinferni et al. (2006) and represent a significant advance over older models. Figure 2 shows the resulting compilation of mass function, $\Psi(M)=\frac{d N}{d M}$. As with the luminosity functions, we have made no attempt to adjust these data for dynamical effects to give estimates of the global present-day mass function.

Stellar mass functions, whether for local disk stars or members of the Galactic halo, are generally characterized either as power laws (sometimes multi-component power laws) or as lognormal distributions (Reid \& Gizis 1997; Silvestri et al. 1998; Piotto \& Zoccali 1999; Kroupa 2002; Chabrier 2003; Cruz et al. 2007; Covey et al. 2008). Paresce \& de Marchi (2000), in particular, have argued that globular cluster luminosity functions are better represented as lognormal distributions. It is important, of course, to recognize that in the absence of an underlying physical theory of star formation, both power laws and lognormal distributions are mathematical representations of the mass function, rather than fundamental descriptors. The principal difference between the two parameterizations is that the lognormal distribution provides a turnover in the mass distribution and a characteristic mass. The latter is typically set at $M_{c} \approx 0.35 M_{\odot}$ $\left(\log \left(M_{c}\right)=-0.46\right.$; Paresce \& de Marchi 2000). Considering the results shown in Figure 2, most clusters have mass functions that are closer in form to a power law than a lognormal distribution. Several show evidence for a flattening in the mass function at low masses, which might be interpreted as a consequence of dynamical evolution and preferential stripping of low-mass 

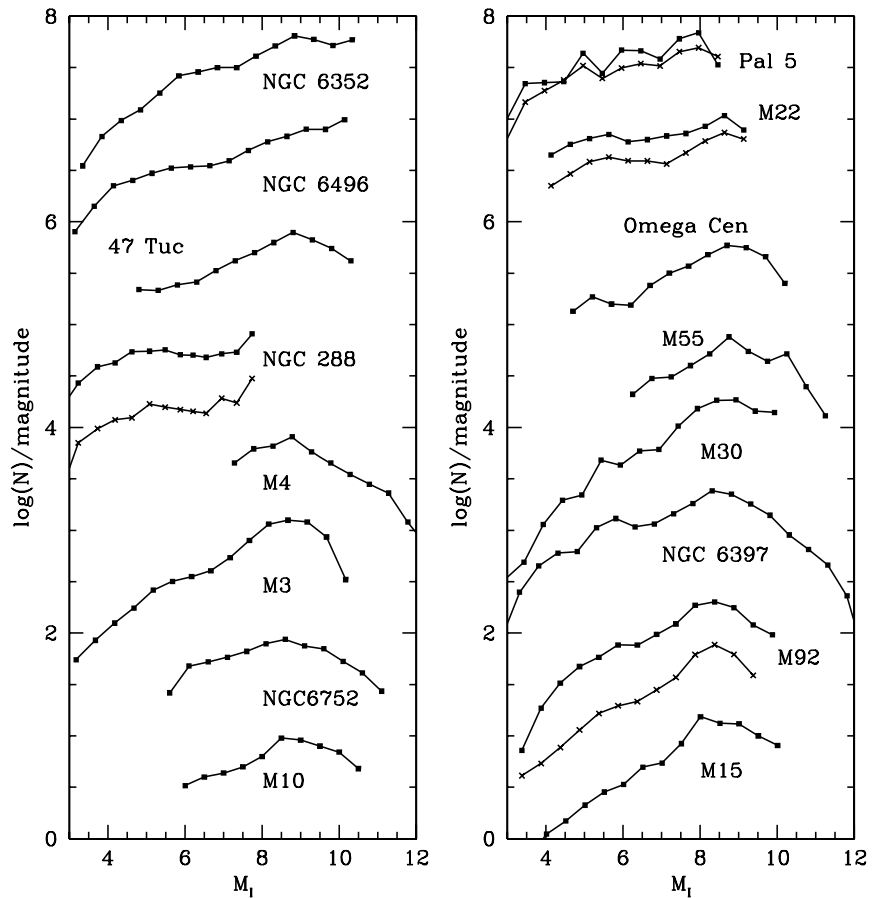

Figure 1. Luminosity functions derived from previous HST observations of the clusters listed in Table 1 . These data, which are plotted with an arbitrary zero point for each cluster, have been transformed to the Cousins $I$ band (generally using the relations in Holtzman et al. 1995) and are taken directly from the published papers. They represent the present-day luminosity functions for the regions surveyed, corrected for photometric incompleteness but with no corrections for dynamical effects. In a few cases (NGC 288, M22, Pal 5, M92), data are provided for two spatially distinct regions within the cluster; we show both data sets here, using crosses to mark data taken at larger radii from the cluster center.
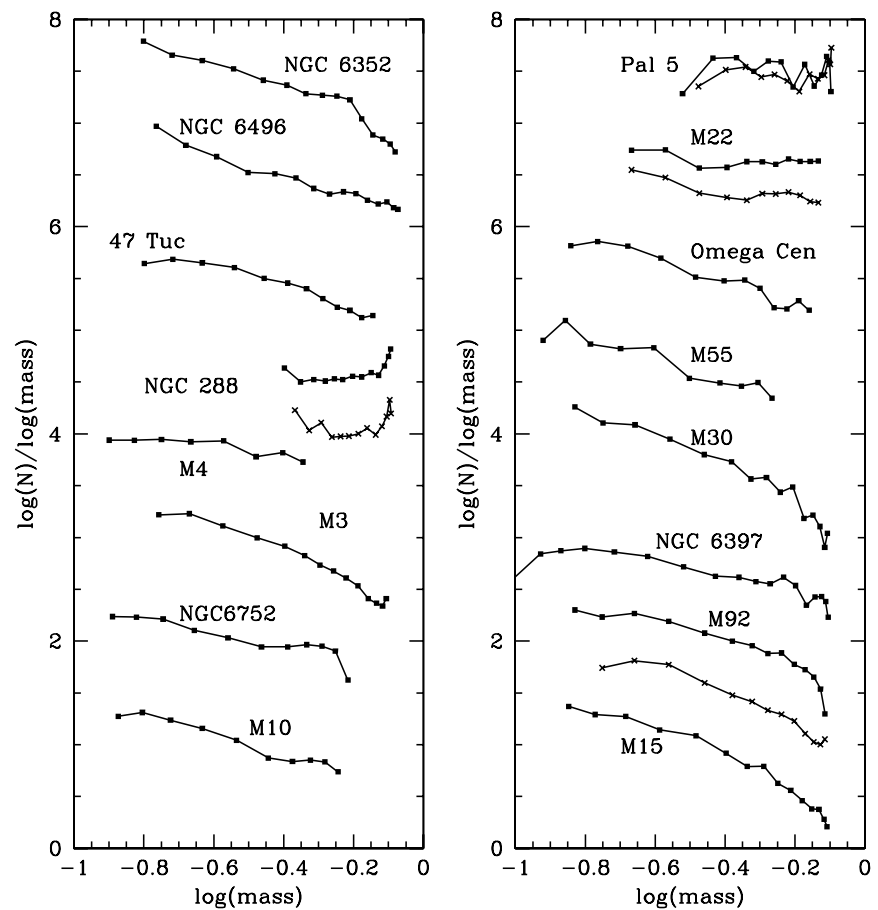

Figure 2. Globular cluster mass functions derived by applying mass-luminosity relations from Dotter et al. (2007) to luminosity functions shown in Figure 1. As with the luminosity functions, the data are plotted with an arbitrary zero point, and these present-day mass functions are not corrected in any respect for dynamical effects such as mass segregation and tidal stripping. The best-fit power laws, on a scale where Salpeter $=2.3$, range from $\alpha \sim-0.3$ for M22 and NGC 288 to $\alpha=2.2$ for M15. As in Figure 1, for clusters with spatially resolved star counts, we use crosses to identify data taken at the larger radii.
Table 2

Cluster Locations

\begin{tabular}{|c|c|c|c|c|c|}
\hline ID & Name & R.A. & Decl. & $l$ & $b$ \\
\hline NGC 104 & 47 Tuc & $00^{\circ} 24^{\prime} 05^{\prime \prime} \cdot 2$ & $-72^{\circ} 04^{\prime} 51^{\prime \prime}$ & 305.90 & -44.89 \\
\hline NGC 288 & & $00^{\circ} 52^{\prime} 47^{\prime \prime} .5$ & $-26^{\circ} 35^{\prime} 24^{\prime \prime}$ & 152.28 & -89.38 \\
\hline NGC 362 & & $01^{\circ} 03^{\prime} 14^{\prime \prime} .3$ & $-70^{\circ} 50^{\prime} 54^{\prime \prime}$ & 301.53 & -46.25 \\
\hline NGC 1261 & & $03^{\circ} 12^{\prime} 15^{\prime \prime} .3$ & $-55^{\circ} 13^{\prime} 01^{\prime \prime}$ & 270.54 & $-52 \circ .13$ \\
\hline NGC 3201 & & $10^{\circ} 17^{\prime} 36^{\prime \prime} .8$ & $-46^{\circ} 24^{\prime} 40^{\prime \prime}$ & 277.23 & 8.64 \\
\hline NGC 5053 & & $13^{\circ} 16^{\prime} 27^{\prime \prime} .0$ & $+17^{\circ} 41^{\prime} 53^{\prime \prime}$ & 335.69 & 78.94 \\
\hline NGC 5272 & M3 & $13^{\circ} 42^{\prime} 11^{\prime \prime} \cdot 2$ & $+28^{\circ} 22^{\prime} 32^{\prime \prime}$ & 42.21 & 78.71 \\
\hline NGC 5286 & & $13^{\circ} 46^{\prime} 26^{\prime \prime} .5$ & $-51^{\circ} 22^{\prime} 24^{\prime \prime}$ & 311.61 & 10.57 \\
\hline NGC 5466 & & $14^{\circ} 05^{\prime} 27^{\prime \prime} \cdot 3$ & $+28^{\circ} 32^{\prime} 04^{\prime \prime}$ & 42.15 & 73.59 \\
\hline NGC 5904 & M5 & $15^{\circ} 18^{\prime} 33^{\prime \prime} .8$ & $+02^{\circ} 04^{\prime} 58^{\prime \prime}$ & 3.86 & 46.80 \\
\hline NGC 5927 & & $15^{\circ} 28^{\prime} 00^{\prime \prime} 5$ & $-50^{\circ} 40^{\prime} 22^{\prime \prime}$ & 326.60 & 4.86 \\
\hline NGC 6093 & M 80 & $16^{\circ} 17^{\prime} 02^{\prime \prime} .5$ & $-22^{\circ} 58^{\prime} 30^{\prime \prime}$ & 352.67 & $19: 46$ \\
\hline NGC 6205 & M13 & $16^{\circ} 41^{\prime} 41^{\prime \prime} .5$ & $+36^{\circ} 27^{\prime} 37^{\prime \prime}$ & 59.01 & 40.91 \\
\hline NGC 6341 & M 92 & $17^{\circ} 17^{\prime} 07^{\prime \prime} \cdot 3$ & $+43^{\circ} 08^{\prime} 11^{\prime \prime}$ & 68.34 & 34.86 \\
\hline NGC 6362 & & $17^{\circ} 31^{\prime} 54^{\prime \prime} 8$ & $-67^{\circ} 02^{\prime} 53^{\prime \prime}$ & 325.55 & -17.57 \\
\hline NGC 6541 & & $18^{\circ} 08^{\prime} 02^{\prime \prime} \cdot 2$ & $-43^{\circ} 30^{\prime} 00^{\prime \prime}$ & 349.48 & -11.09 \\
\hline NGC 7099 & M 30 & $21^{\circ} 40^{\prime} 22^{\prime \prime} .0$ & $-23^{\circ} 10^{\prime} 45^{\prime \prime}$ & 27.18 & -46.83 \\
\hline
\end{tabular}

Note. All data taken from Harris (1996).

subdwarfs, but few show a distinct turnover near favored values of $M_{c}$.

The imaging data obtained in the course of the ACS GGC survey have sufficient sensitivity to reach $\approx 0.3 M_{\odot}$ MS stars in most clusters at the $50 \%$ completeness limit, but only extend significantly below $M_{c}$ in a few cases. However, the areal coverage provided by ACS allows us to map the degree of mass segregation and use King models to apply corrections to derive the global present-day luminosity function. Given these constraints, we have chosen to characterize the derived mass functions using the power-law formalism, since this provides a simple method of correlating the results against other intrinsic or extrinsic cluster parameters. Extending these observations to fainter magnitudes in more clusters will provide deeper insight into the prevalence of lognormal mass functions.

\section{PHOTOMETRY AND COMPLETENESS}

Observations for this work were collected using the WFC on the ACS aboard the HST as part of the HST/ACS Survey of GGCs. We have observations of 65 clusters (although only partial data for one, NGC 5897). In a previous paper (Paust et al. 2009, Paper VI), we discussed the luminosity and mass function for NGC 6366, a metal-rich member of the Bulge population. Here we use the same techniques to analyze data for the 17 clusters listed in Table 2. Those clusters were chosen to be representative of the larger sample and cover a range of locations in the Galaxy as well as spanning a range of physical sizes, metallicities, and ages. Several clusters have moderate foreground reddening, but all have relatively narrow color-magnitude diagrams (CMDs), indicating a low level of differential reddening across the ACS field of view. Analyses of the full sample will be presented in a later paper in this series.

The same observing methodology was used for all the clusters observed (see Paper VI for full details). The WFC, with an approximately 3 arcmin field of view, was centered on the cluster core for each cluster. One short exposure, to avoid saturating the red giant branch $(\mathrm{RGB})$ and four to five longer exposures were taken in the F606W and F814W filters with a total of one orbit of exposure time used in each filter. The primary goal, as outlined in Sarajedini et al. (2007) was to obtain good photometry from the level of the horizontal branch down to approximately $0.2 M_{\odot}$ in 
each cluster. In the more distant and/or highly reddened clusters, the lower mass limit is closer to $\approx 0.35 M_{\odot}$.

To this end, an elaborate method was devised for automatic finding and photometric measurement of the images of each cluster Anderson et al. (2008). The method relies on a number of principles and procedures, which are too elaborate and complex to describe in detail here. A few of its salient points are: (1) all finding procedures and measurements are done on the $f l t$ images (bias-corrected and flat-fielded but not processed in any other way), rather than on drizzled images, since drizzling distorts the stellar profile and washes out the photometric and astrometric information. (2) The finding procedures were designed to search all the flt images simultaneously in order to identify objects that fell in the same place in the field in multiple dithered exposures. (3) The field was searched one small patch at a time; the patch was large enough to allow us to deal with nearby-neighbor effects, but small enough that the PSF and distortion could be treated as constant within the patch. (4) An accurate PSF model (Anderson \& King 2006) is constructed for each star for each exposure, based on the location of the star in the chip and the variation in focus that comes from breathing. (5) Star positions and fluxes were measured by least-squares fitting of the PSF to the innermost pixels of each star in each exposure, after removing the contributions of any nearby neighbor. (6) The output of the procedure is a list of stars in the field with average positions and fluxes, and estimates of errors in these averages based on the agreement among the individual exposures. The routine also produced some quality diagnostics that can be used to identify the best-measured stars. (7) Artificial-star tests were performed in an identical way to the real-star measurements, by placing test stars at the same place in the field in each exposure and with a flux that put them on the fiducial cluster sequence. The same finding and measuring procedures that were used for the real stars were used to report which artificial stars were found, and how well.

Representative CMDs for five clusters, covering a broad range of metallicity, are shown in Figures 3-7. Table 3 tabulates the estimated ages, distance moduli, and color excesses derived from the isochrone fits for the full sample of clusters discussed in the present paper. In fitting the isochrones, the cluster metallicities were initially assumed to be the Harris (1996) values. Those values were adjusted until the observed and model RGB slopes matched. In most cases, the resulting bestfit metallicity is close to the Zinn \& West (1984) value. We also list the standard values for apparent distance moduli and reddening from Harris (1996). Adopting standard reddening curves $\left(E_{V-I}=1.25 E_{B-V}\right)$, our results are in good agreement with the literature data with the exception of NGC 288. The isochrone fitting is discussed in more detail in Section 5.1, where also consider the NGC 288 result and the implications for determining the mass function.

The photometric completeness for each cluster was determined through artificial star tests. For each cluster, a total of $10^{5}$ artificial stars were added sampling the entire area of the images. The input stars were given realistic uncertainties upon insertion into the image. Those stars were recovered using the same patch-based finding and measuring method as used for real stars. A unique feature of this approach is that artificial stars are added individually, one at a time, instead of in sets, as is more typical for other software programs. As a result, the artificial stars do not crowd and interfere with each other resulting in an excellent representation of the true completeness of the photometry. Artificial stars were considered to be recovered if they were

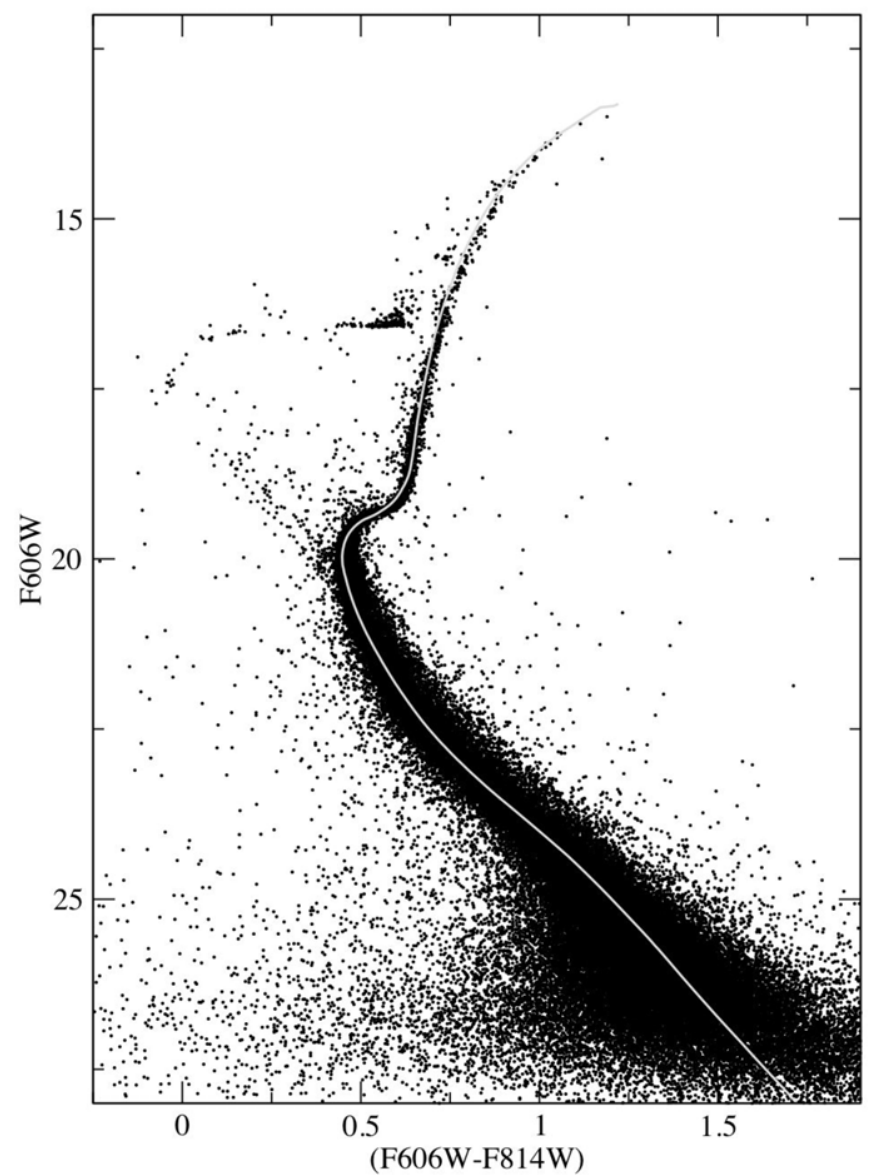

Figure 3. CMD for NGC 1261. The observations are fit with a $[\mathrm{Fe} / \mathrm{H}]=-1.35$ $12.0 \mathrm{Gyr}$ isochrone with an apparent distance modulus of 16.15 and a color excess of 0.03 .

found within 0.75 pixels of their input position and $0.75 \mathrm{mag}$ of their input magnitude. Those criteria prevent nearby real stars from causing false-positive recoveries of the artificial stars.

Completeness corrections for each cluster are handled in the same manner as in Paust et al. (2007). Two parameters are used to interpret the artificial star results: distance from the cluster center, which is directly related to the degree of crowding, and magnitude. Based on the detection or nondetection of individual artificial stars, we formed a grid of completeness $(R, m)$ with values at each point ranging from 0.0 where all the artificial stars were lost to 1.0 where all the artificial stars were recovered. Using this grid, real stars from the photometric catalogue were then assigned weights of completeness ${ }^{-1}(R, m)$ with values interpolated to the true magnitude and core distance for each star. This method greatly simplifies the creation of luminosity and mass functions in specific areas of the cluster; summing the weights of the stars in that region gives the completeness-corrected star counts.

A map of completeness in M92 as a function of magnitude and radius from the cluster center can be seen in Figure 8. The photometry has greater than $50 \%$ completeness across the majority of the parameter space, only dipping below this level for faint stars near the cluster center where crowding becomes a significant issue. Other clusters show a similar pattern although the exact location of the various contours depends strongly on the degree of crowding. All of the clusters in this work have very crowded cores, and the completeness drops below $25 \%$ within those regions for stars along the lower MS. In our analyses, we 
Table 3

Derived Cluster Parameters

\begin{tabular}{ccccccccccccc}
\hline \hline ID & $(m-M)_{606}$ & $E_{606-814}$ & $(m-M)_{V}$ & $E_{B-V}$ & $\begin{array}{c}\text { Age } \\
(\mathrm{Gyr})\end{array}$ & {$[\mathrm{Fe} / \mathrm{H}]_{\mathrm{fit}}$} & {$[\mathrm{Fe} / \mathrm{H}]_{\mathrm{ZW}}$} & {$[\mathrm{Fe} / \mathrm{H}]_{\mathrm{GC}}$} & $\begin{array}{c}R_{\text {core }} \\
(\operatorname{arcmin})\end{array}$ & $\begin{array}{c}R_{\mathrm{t}} \\
(\operatorname{arcmin})\end{array}$ & $\begin{array}{c}c \\
\text { PDMF Slope }\end{array}$ \\
\hline NGC 104 & 13.50 & 0.05 & 13.37 & 0.04 & 12.5 & -0.75 & -0.71 & -0.78 & 0.67 & 16.25 & 1.38 & $-0.84 \pm 0.12$ \\
NGC 288 & 14.90 & 0.18 & 14.83 & 0.03 & 13.0 & -1.20 & -1.40 & -1.14 & 1.41 & 12.94 & 0.96 & $-0.83 \pm 0.07$ \\
NGC 362 & 14.80 & 0.04 & 14.81 & 0.04 & 12.0 & -1.25 & -1.33 & -1.09 & 0.17 & 13.5 & 1.90 & $-1.69 \pm 0.06$ \\
NGC 1261 & 16.15 & 0.03 & 16.10 & 0.04 & 12.0 & -1.35 & -1.32 & -1.09 & 0.41 & 6.2 & 1.18 & $-0.59 \pm 0.04$ \\
NGC 3201 & 14.20 & 0.30 & 14.21 & 0.21 & 12.0 & -1.58 & -1.53 & -1.24 & 1.23 & 28.45 & 1.36 & $-0.77 \pm 0.05$ \\
NGC 5053 & 16.30 & 0.04 & 16.19 & 0.01 & 12.0 & -1.99 & -2.10 & -1.98 & 1.48 & 13.4 & 0.96 & $-1.46 \pm 0.22$ \\
NGC 5272 & 15.15 & 0.04 & 15.12 & 0.01 & 12.0 & -1.57 & -1.66 & -1.34 & 0.45 & 14.19 & 1.50 & $-1.31 \pm 0.11$ \\
NGC 5286 & 16.00 & 0.28 & 15.95 & 0.24 & 13.0 & -1.67 & -1.70 & -1.41 & 0.34 & 8.36 & 1.39 & $-0.32 \pm 0.02$ \\
NGC 5466 & 16.20 & 0.05 & 16.00 & 0.00 & 12.0 & -2.22 & -2.22 & -2.20 & 1.41 & 14.2 & 1.00 & $-1.15 \pm 0.03$ \\
NGC 5904 & 14.50 & 0.06 & 14.46 & 0.03 & 12.0 & -1.37 & -1.38 & -1.12 & 0.60 & 13.4 & 1.35 & $-1.15 \pm 0.09$ \\
NGC 5927 & 16.00 & 0.45 & 15.81 & 0.46 & 11.0 & -0.70 & -0.32 & -0.62 & 0.55 & 32.8 & 1.78 & $-1.44 \pm 0.11$ \\
NGC 6093 & 15.80 & 0.24 & 15.56 & 0.20 & 13.0 & -1.75 & -1.75 & -1.47 & 0.18 & 7.1 & 1.60 & $-1.36 \pm 0.10$ \\
NGC 6205 & 14.50 & 0.04 & 14.35 & 0.02 & 13.0 & -1.60 & -1.63 & -1.33 & 0.76 & 16.2 & 1.33 & $-0.98 \pm 0.02$ \\
NGC 6341 & 14.75 & 0.05 & 14.64 & 0.02 & 13.5 & -2.16 & -2.24 & -2.16 & 0.25 & 15.2 & 1.78 & $-1.23 \pm 0.08$ \\
NGC 6362 & 14.70 & 0.09 & 14.67 & 0.10 & 14.0 & -1.08 & -1.18 & -0.99 & 1.20 & 17.7 & 1.17 & $-0.49 \pm 0.04$ \\
NGC 6541 & 14.80 & 0.15 & 14.67 & 0.13 & 13.5 & -1.85 & -1.79 & -1.53 & 0.22 & 22.6 & 2.01 & $-1.07 \pm 0.07$ \\
NGC 7099 & 14.75 & 0.07 & 14.62 & 0.05 & 13.0 & -2.12 & -2.05 & -1.99 & 0.04 & 14.3 & 2.55 & $-0.92 \pm 0.06$ \\
\hline
\end{tabular}

Notes. The $V$-band apparent distance moduli and $E_{B-V}$ values are taken from Harris (1996), and the metallicities on the GC (Gratton \& Carretta) and ZW (Zinn \& West) scales are taken from Rutledge et al. (1997).

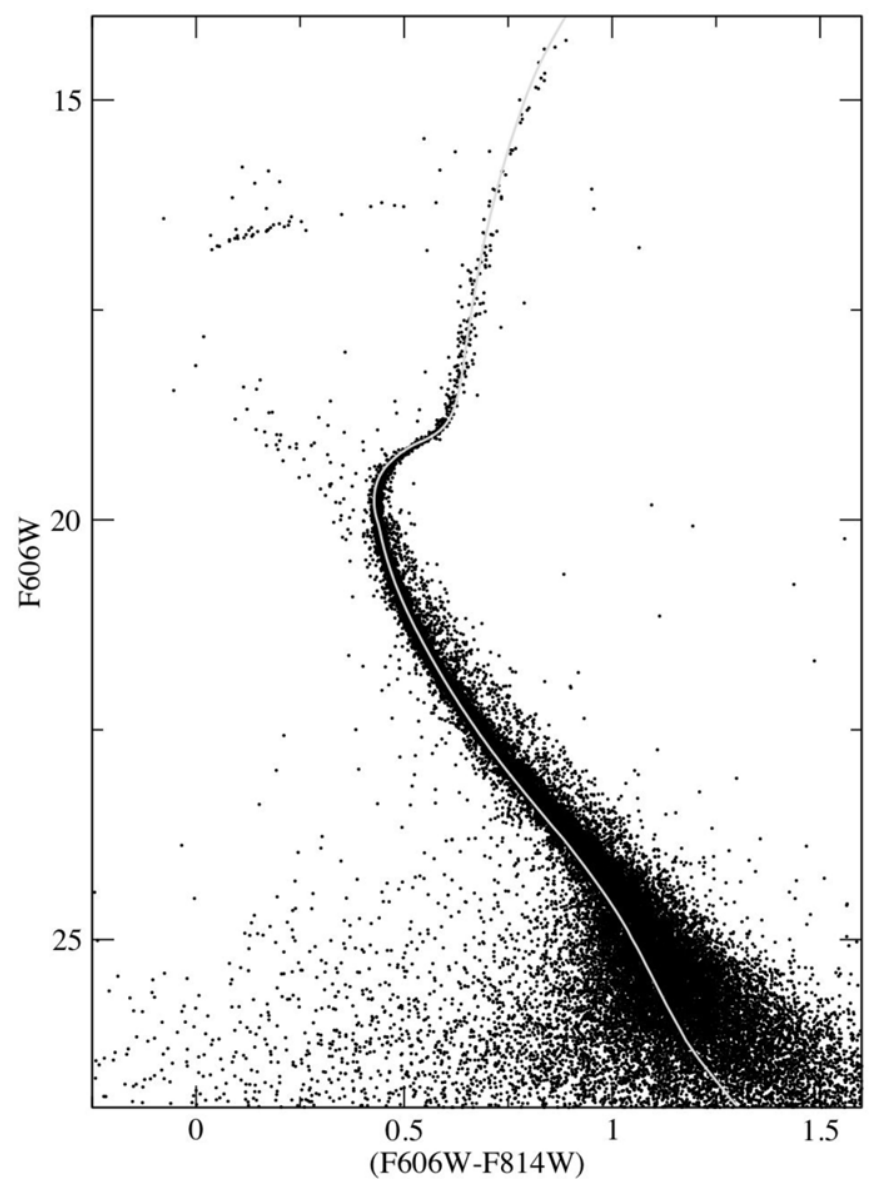

Figure 4. CMD for NGC 5466. The observations are fit with a $[\mathrm{Fe} / \mathrm{H}]=-2.22$ 12.0 Gyr isochrone with an apparent distance modulus of 16.20 and a color excess of 0.05 .

have excluded regions that are less than $50 \%$ complete, that is, where the corrections become larger than a factor of two. As a result, we do not include in our analyses the central $\sim 25$ arcsec of the clusters considered here, since the data sets reach that level of incompleteness for most of the lower MS.

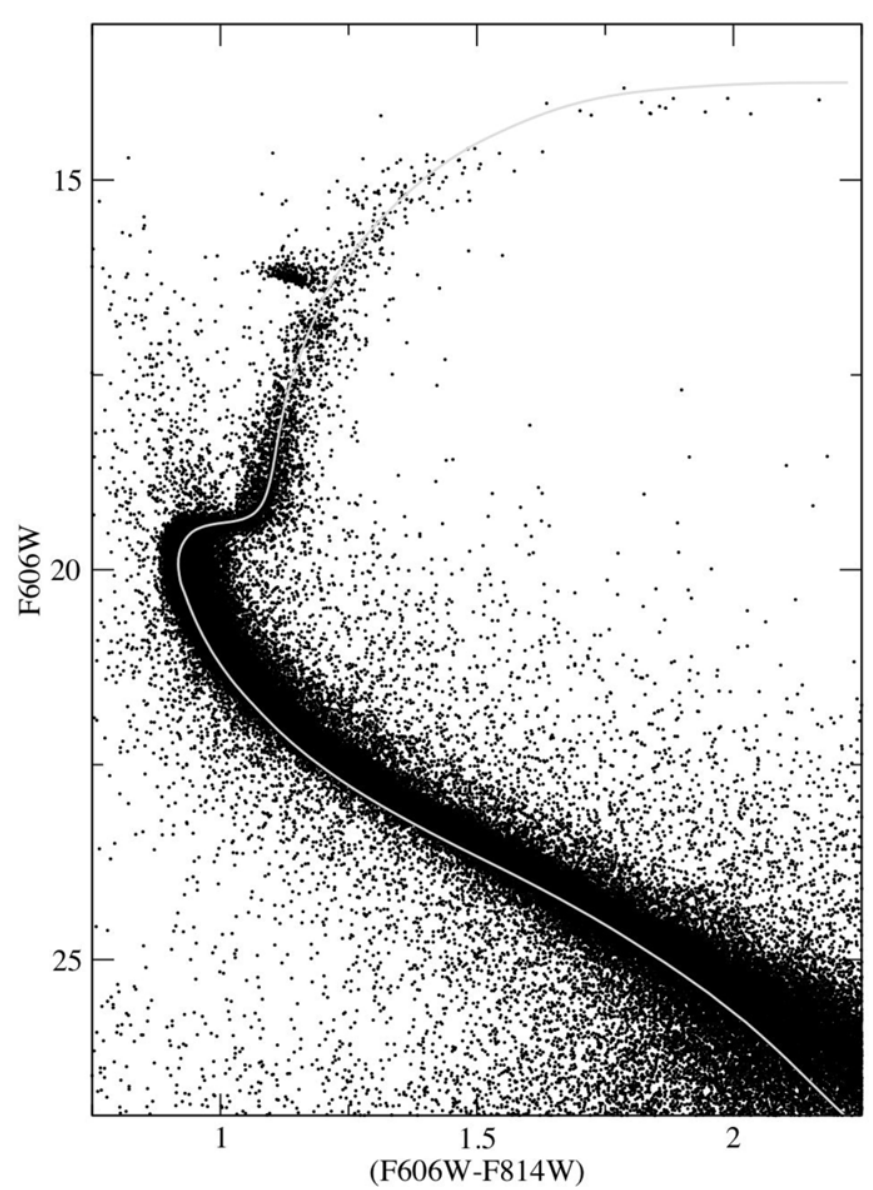

Figure 5. CMD for NGC 5927. The observations are fit with a $[\mathrm{Fe} / \mathrm{H}]=-0.70$ $11.0 \mathrm{Gyr}$ isochrone with an apparent distance modulus of 16.00 and a color excess of 0.45 .

\section{DYNAMICAL MODELING}

The ACS field of view is very limited when compared to overall angular diameter of the clusters under study. With the core centered in an image, it is only possible to observe to a 


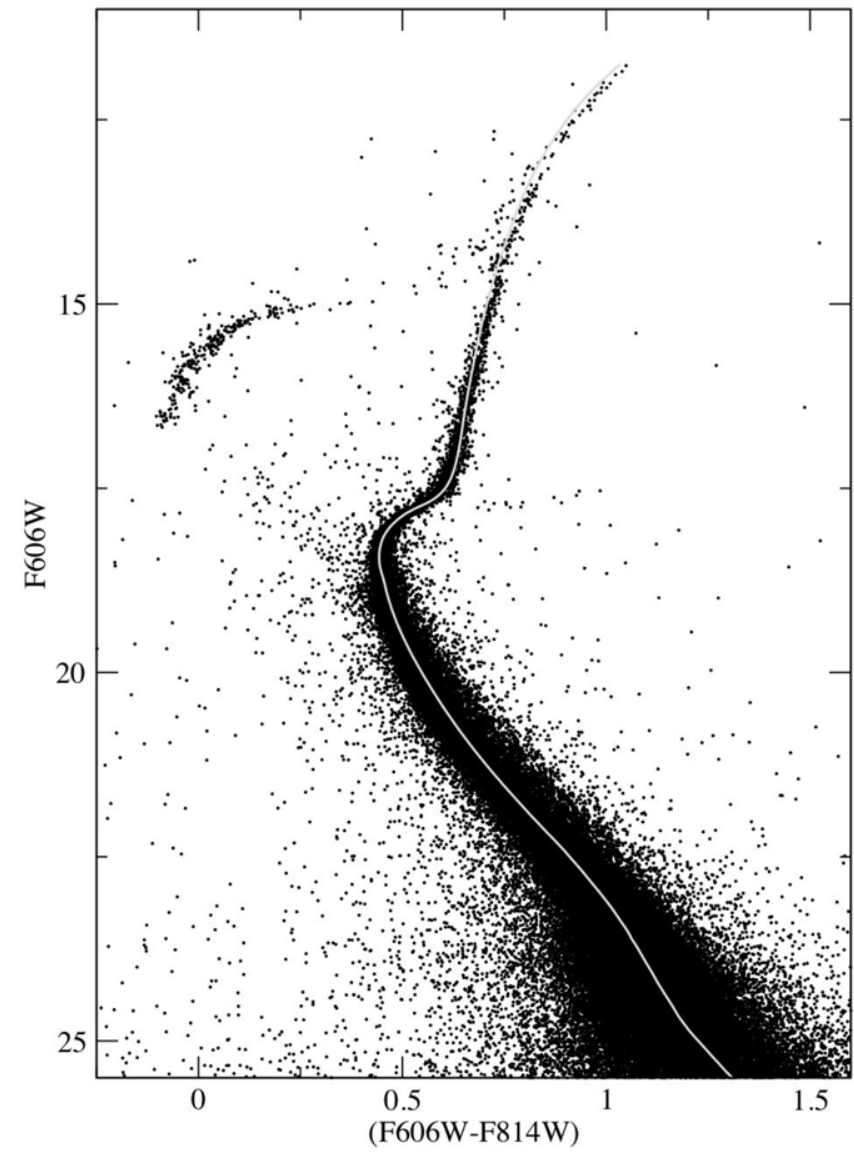

Figure 6. CMD for NGC 6341 (M92). The observations are fit with a $[\mathrm{Fe} / \mathrm{H}]=-2.1613 .5 \mathrm{Gyr}$ isochrone with an apparent distance modulus of 14.75 and a color excess of 0.05 .

radius of approximately 1.83 arcmin while the tidal radius of a cluster could be over 30 arcmin. As a result, it is necessary to extrapolate our local observations to the global behavior of the cluster while taking mass segregation into account. To accomplish this, multi-mass King models are used. The specific King-model code used in this work was developed by Anderson (1997), and is taken directly from the formalism described in Gunn \& Griffin (1979). The code represents the cluster as a set of population groups. Stars within each group are characterized by the same mass and respond to the potential induced by the sum of all the groups.

In order to properly constrain a multi-mass King model, we must specify the contribution of stars at a particular mass (the population groups) either in terms of the total mass, or in terms of the mass fraction at a given radius. In addition, we must provide some global cluster parameters, including the distance, core radius and tidal radius. Since our prime concern is determining how the LF varies with radius, it is convenient to associate the population groups with bins in the LFs, assigning each group the average mass for stars in that LF bin.

We adopted the annulus between 25 and 50 arcsec as the region over which we constrained our model LF to fit the observations. This region provides the reference luminosity function. The chosen location represents a balance between the desire for a large number of stars and low Poisson statistics, and the need for a narrow annulus to minimize variations in the intrinsic structural parameters of the clusters.

In creating the input luminosity function, the RGB and MS were binned in $1 \mathrm{mag}$ bins to represent the luminosity function.

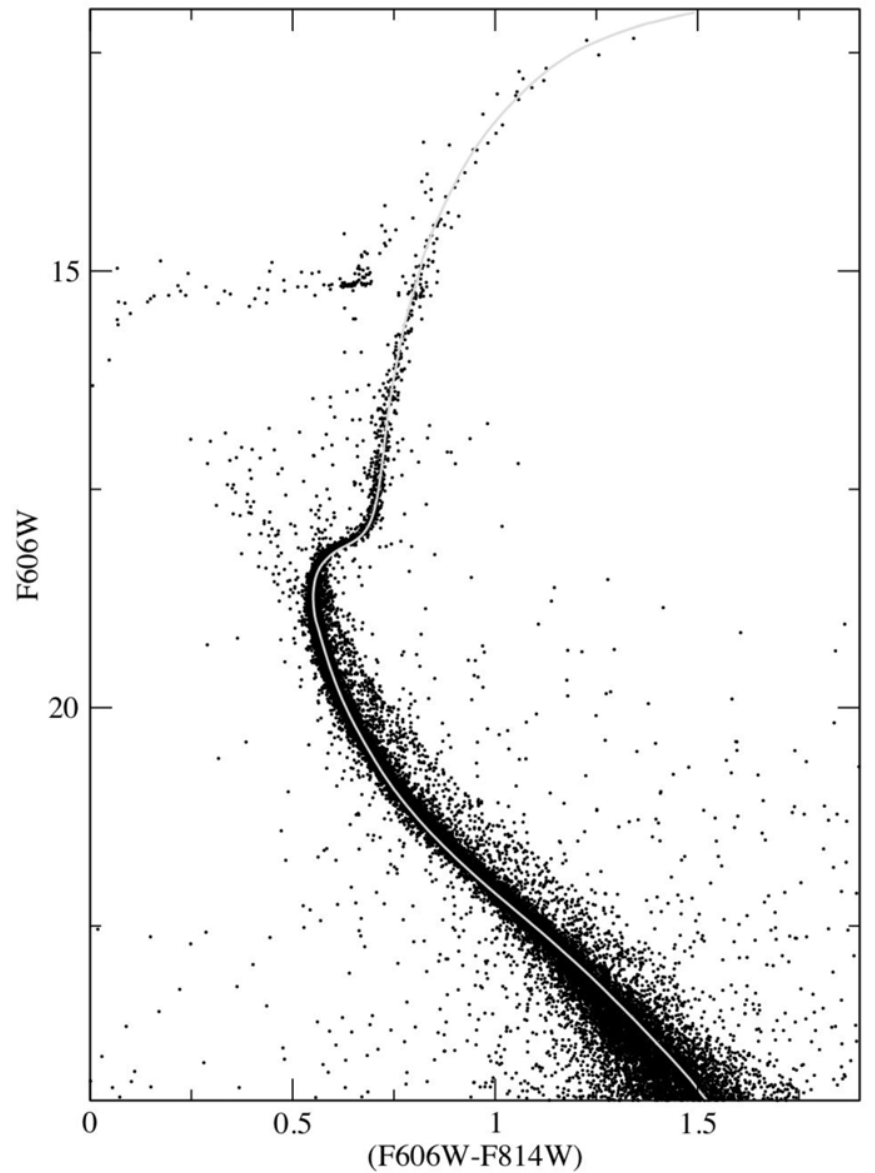

Figure 7. CMD for NGC 6362. The observations are fit with a $[\mathrm{Fe} / \mathrm{H}]=-1.08$ 14.0 Gyr isochrone with an apparent distance modulus of 14.70 and a color excess of 0.09 .

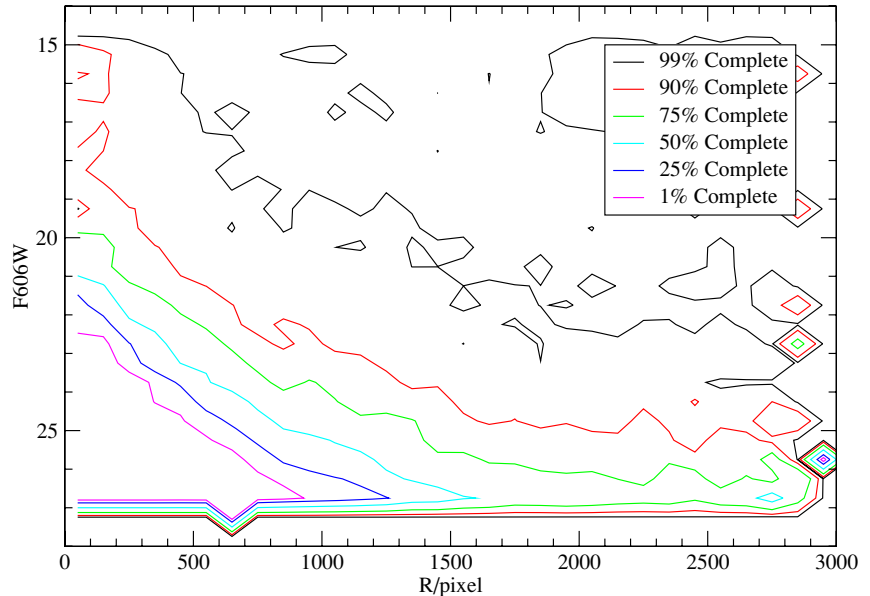

Figure 8. Completeness map for M92 showing the completeness determined from artificial star tests as a function of magnitude and radius from the cluster center. The photometry has very good completeness except for faint stars near the center of the cluster, as seen in the bottom left of the plot.

Each bin corresponds to a group of stars with the appropriate mass in the dynamical models. Additional mass groups were added to represent stars on the horizontal branch and asymptotic giant branch, blue stragglers, and compact objects in the form of neutron stars and white dwarfs. In a cluster, which has not been subjected to significant stripping, the expected population of neutron stars and white dwarfs can be determined a priori. The numbers of neutron stars and white dwarfs were estimated assuming a power-law IMF with an index of -1.0 for stars 
between the AGB tip and $1 M_{\odot}$ (broadly consistent with the results plotted in Figure 2) and an index of -2.3 for stars above that mass. Stars that formed with masses between $0.8 M_{\odot}$, the mass of stars on the RGB, and $6.5 M_{\odot}$ were assigned to be white dwarfs while higher mass stars were assumed to have evolved into neutron stars. Integrating this assumed IMF at high masses, one would expect a globular cluster to contain $0.5 \%$ neutron stars (by number) at $1.5 M_{\odot}$ and $15 \%$ white dwarfs (by number) at $0.6 M_{\odot}$. We have adopted mass-luminosity relationships from the DSEP models of Dotter et al. (2007) after a thorough evaluation (Paust et al. 2009).

The observed structural parameters for each cluster, the core and tidal radii, deserve special mention. The published values for the core radius taken from the Harris (1996) catalog, for example, are largely based on surface brightnesses derived from ground-based star counts. The extreme resolving power of the $H S T$ and the high quality of our photometry allow us to refine the measured core radius. Thus, core radii derived from our photometric catalogues were used in the King models. Our refined core radii are typically within $10 \%$ of the published values from Harris (1993).

Conceptually, the tidal radius gives the radius to which cluster stars are gravitationally bound. However, the stellar density is so low at that distance that it is not possible to observationally measure the tidal radius without multiple epochs of deep widefield imaging. Those data would allow low-mass, faint cluster members, which are expected to dominate the population of cluster stars at large radii, to be separated from field stars based on their proper motions. Such observations are not yet available for any clusters in the present sample and as a result, the tidal radius derived here is derived by fitting the concentration to the profile from the inner annuli. In some cases (e.g., NGC 104, 47 Tuc) the values derived in this analysis differ significantly from the standard parameters.

As an additional complication, uncertainties in the surface brightness profile at large radii are such that the tidal radius can vary significantly without affecting the agreement between the model and observed surface brightnesses. To the extent that data are available, we use the Trager et al. (1995) surface brightness profiles for comparison with our models although these profiles typically only extend to a fraction of the tidal radius. The Trager et al. (1995) profiles are converted from $V$ magnitudes to $F 606 \mathrm{~W}$ using the Sirianni et al. (2005) transformations using the average color of stars brighter than the MSTO. Over $80 \%$ of the light in the surface brightness profile comes from stars brighter than the MSTO and, as a result, this transformation works well.

On the other hand, the dominant role of MSTO stars means that the surface brightness profile characterizes the spatial distribution of stars drawn from a relatively small mass interval at the upper end of the mass function. The results take no account of mass segregation and the more extended distribution of lower mass stars. As a result, the profile provides a relatively weak constraint on the tidal radius. Stellar number counts can supply stronger constraints, as discussed recently by Bonatto \& Bica (2008). We have used the ACS data to that effect. We were able to iterate on an appropriate tidal radius for each cluster by examining the agreement between the observed and model luminosity functions as a function of radius within the cluster. The final tidal radii often differ from the values listed in the Harris catalog by over $20 \%$.

A full description of the modeling can be found in Paust et al. (2009). The process can be simply described by the following four-step process.
1. Using the cluster stellar density profile, the core radius, defined as the HWHM of the stellar density profile for stars brighter than the MSTO, is derived. The tidal radius is initially taken from the Harris (1996) catalog.

2. A local luminosity function for the annulus from 25 to 50 arcsec is generated. A mass-luminosity relationship is used assign a mass to each bin in the luminosity function, and each set of stars is associated with a mass group in the multimass King model. Additional mass groups are included in the model to represent neutron stars and white dwarfs.

3. The model output is then used to produce several data products: a model surface brightness profile and model luminosity functions in annuli from 25 to 50,50 to 75 , and 75 to 110 arcsec. The surface brightness profile is compared to the ACS/WFC surface brightness profile which is extended to larger radii using data from Trager et al. (1995). The model LFs in each annulus are compared to the observed LFs.

4. The tidal radius is adjusted and the King model is run again until the observed and model surface brightness profiles match and the model LFs have the same slope. Even the Trager et al. (1995) profiles do not extend to the full tidal radius for most clusters, so the surface brightnesses are a somewhat weak constraint on the actual tidal radius. Comparison of the observed and predicted LFs as a function of radius provides a means of verifying the solution, since changing the tidal radius affects the predicted degree of mass segregation.

It is important to note that the only free parameter in this process is the tidal radius. The core radius is constrained by the stellar density profile and the input luminosity function is constrained directly by our observations.

\section{THE CLUSTERS}

\subsection{Isochrone Fitting, Ages, and Distances}

Transforming the observed number-magnitude counts for each cluster to, first, a luminosity function and then a mass function requires that we estimate the distance, and then apply the appropriate mass-luminosity relation. We have used theoretical models from the Dartmouth Stellar Evolution Program (DSEP; Dotter et al. 2007) to derive those parameters. As discussed in Paust et al. (2009), the DSEP stellar evolution models are in excellent agreement with other state of the art models, notably by Montalban et al. (2000) and Pietrinferni et al. (2006). Distances are determined through isochrone fitting against the observed $\mathrm{CMD}$, and the corresponding mass-magnitude relation used to convert the observed luminosity functions to mass functions.

All of the clusters included in this paper have multiple abundance measurements (Zinn \& West 1984; Carretta \& Gratton 1997; Rutledge et al. 1997; De Angeli et al. 2005), and the derived values sometimes span a range of $0.2-0.3 \mathrm{dex}$ for a given cluster. In part, this dispersion reflects the different techniques used for abundance estimation; in particular, Zinn \& West (1984) estimate global metallicities from low-resolution spectra and narrowband photometric (Ca II K line) indices, while Carretta \& Gratton (1997) and Rutledge et al. (1997) use higher resolution spectroscopic observations of individual stars (primarily giants) to derive iron and calcium abundance, respectively. As halo objects, globular clusters are expected to have enhanced abundances of $\alpha$ elements $(\mathrm{Ca}, \mathrm{Mg}$, Ti) relative to iron. Salaris et al. (1993) give the following prescription for determining the effective metallicity, $Z=Z_{0}\left(0.638 f_{\alpha}+0.362\right)$, 


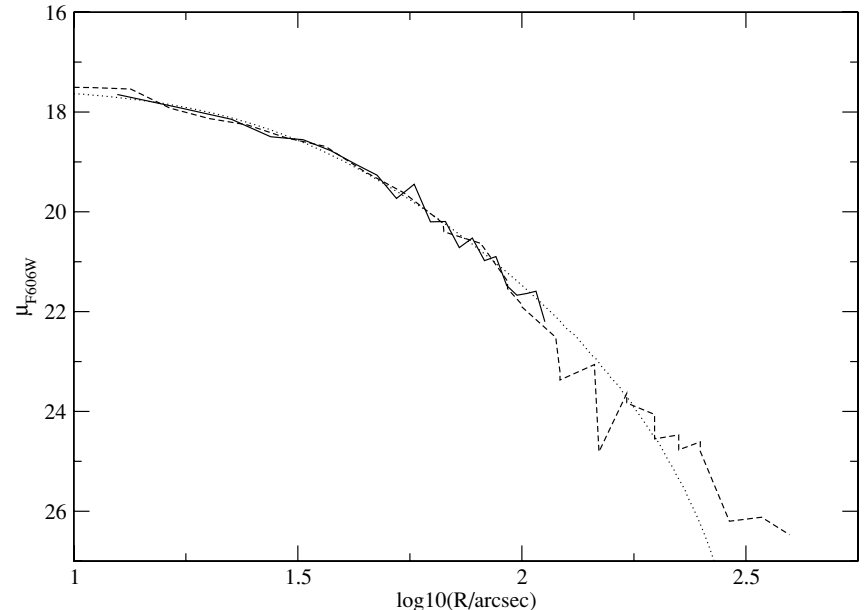

Figure 9. Surface brightness profile for NGC 1261. The dotted line is the surface brightness from our King model, the dashed line is the observation from Trager et al. (1995), and the solid line is the surface brightness derived from our ACS photometry.

where $Z_{0}$ is the iron abundance and $f_{\alpha}$ is the $\alpha$-element enhancement; for $[\alpha / \mathrm{Fe}]=0.3,[m / \mathrm{H}] \sim[\mathrm{Fe} / \mathrm{H}]+0.2 \mathrm{dex}$. Thus, one might expect the global Zinn-West scale to be systematically metal-rich compared with Carreta \& Gratton's analysis; in fact, the situation is reversed, with $[\mathrm{Fe} / \mathrm{H}]_{\mathrm{CG}}$ typically $0.2-0.3$ dex higher than $[\mathrm{Fe} / \mathrm{H}]_{\mathrm{ZW}}$.

Fortunately, the continuing uncertainty in the absolute globular cluster metallicity scale is not of critical importance for analysis of the stellar mass function: the mass-luminosity relation is only weakly dependent on metallicity within the range considered for each cluster. For internal consistency in the present work, we use theoretical isochrones based on scaled-solar metallicity models, and adopt the metallicity and age that gives the best match to the observed CMDs. The derived metallicities, distance moduli and ages are listed in Table 3. For comparison, we list the cluster metallicities on the Zinn/West and Carreta/ Gratton scales, as tabulated by Rutledge et al. (1997). As noted in Section 3, the metallicities derived from DSEP isochrone fitting generally lie closer to the Zinn/West values.

The formal uncertainties in matching against the theoretical isochrones are small: better than 0.05 mag in distance modulus, and $<1 \mathrm{Gyr}$ in age. However, a more realistic estimate must take into account systematic uncertainties in the abundance scale (and hence the choice of isochrones) and, to a lesser extent, in the foreground reddening. Taking those additional factors into account, we estimate that the cluster distance moduli have average uncertainties of $0.15-0.2 \mathrm{mag}$ or $7 \%-10 \%$ in distance. Since $L \propto M^{2}$ for lower-mass stars, this corresponds to uncertainties of $<5 \%$ in the individual masses, and uncertainties of $\sim 5 \%$ in the slope of the best-fit power law.

Systematic errors can also be introduced through the isochrone fitting process. The most extreme case is NGC 288, where we derive substantial foreground reddening. The best-fit metallicity is also closer to the $\mathrm{CG}$ value than the $\mathrm{ZW}$ value, in contrast to most other clusters. This cluster lies near the South Galactic Pole, and our reddening value is clearly an overestimate. However, while our estimated reddening corresponds to a true distance modulus $\sim 0.3$ mag shorter than more traditional values, the corresponding offset in the mass function is only $\sim 8 \%$, or $\sim 0.06 M_{\odot}$ at the turnoff. As a consequence, even systematic errors of this scale have only a minor effect on the mass function.

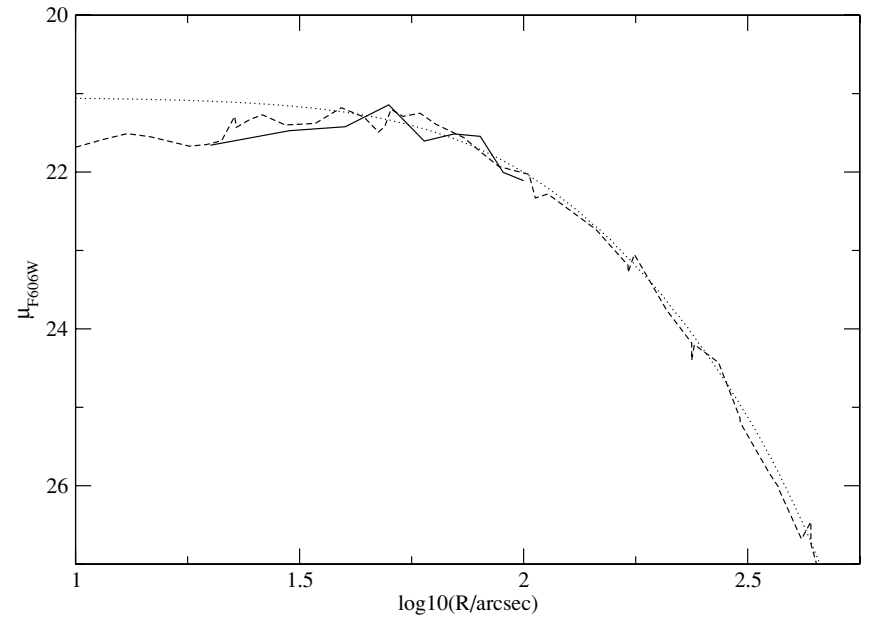

Figure 10. Surface brightness profile for NGC 5466. The dotted line is the surface brightness from our King model, the dashed line is the observation from Trager et al. (1995), and the solid line is the surface brightness derived from our ACS photometry.

\subsection{Generating Global Luminosity and Mass Functions}

A full description of the procedures we use to derive cluster mass functions is given in our previous analysis of NGC 6366 (Paust et al. 2009, Paper VI). Here, we summarize the main points of that analysis. As discussed in Section 4, direct star counts from the ACS data measure the present-day luminosity function within the clusters' central regions. We use multi-mass King models to correct those for mass segregation and derive the global luminosity function. We constrain the King models by specifying a reference LF from a specific annulus. We have adopted the luminosity function from the $25-50$ arcsec annulus as our default input as it covers a fairly narrow radial range and contains the largest number of stars in the majority of our clusters.

Figures 9-13 show the predicted and observed surface brightness profiles for five representative clusters from the present sample, and Figures 14-18 compare the local luminosity functions (as a function of radius) against the King model predictions for the same representative subsample. In all cases, it is clear that the King models accurately track the observed cluster behaviors. For clarity, in the LF figures the observed and model LFs from 50 to 75 arcsec are not shown, however, they agree to the same degree as the 75-110 arcsec data.

Having confirmed that the dynamical models match the observations, those models allow us to extrapolate the "local" observations to create the global present-day luminosity functions. As the clusters in this study are fairly distant, the ACS/WFC field of view captures a significant fraction of the cluster stars. Taking NGC 1261 as an example, the model indicates that the ACS observations encompass nearly $75 \%$ of the cluster stars near the MSTO and approximately $65 \%$ of stars lower on the MS. Given that the higher mass stars are expected to be more centrally concentrated, these results are reasonable.

The global luminosity functions are converted to mass functions using mass-luminosity relations of the appropriate metallicity from the Dotter et al. (2007) models. As discussed in Paust et al. (2009), evolved stars on the subgiant and RGB span a small range in mass, and we truncate the mass function calculations at the MSTO. CMDs for several clusters, including NGC 5466 and NGC 6362 (Figures 3 and 7), reveal clear evidence for binaries on the MS. We include those stars in our analysis, but have not 


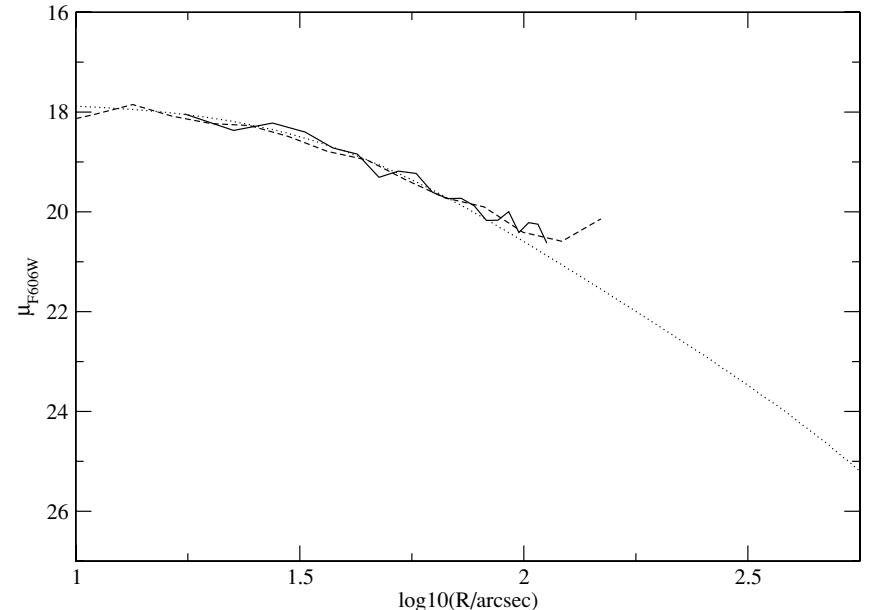

Figure 11. Surface brightness profile for NGC 5927. The dotted line is the surface brightness from our King model, the dashed line is the observation from Trager et al. (1995), and the solid line is the surface brightness derived from our ACS photometry.

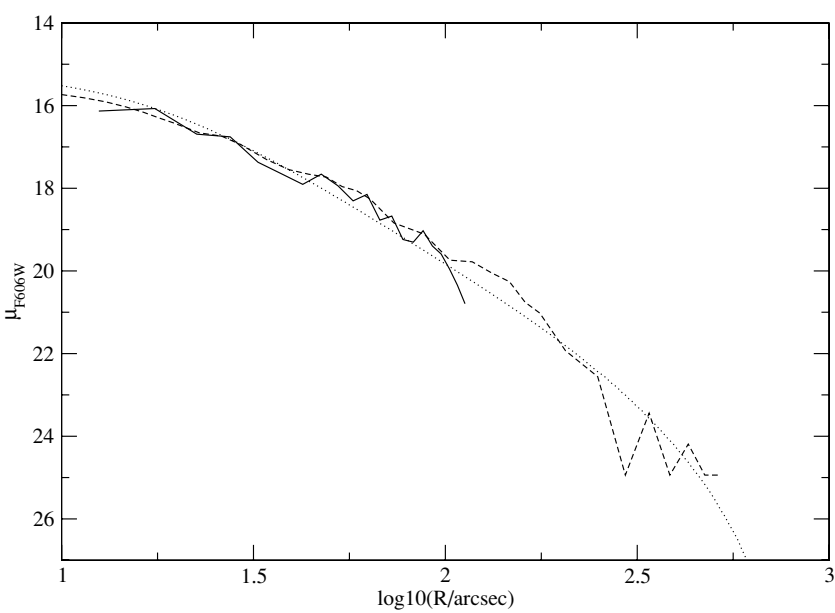

Figure 12. Surface brightness profile for NGC 6341. The dotted line is the surface brightness from our King model, the dashed line is the observation from Trager et al. (1995), and the solid line is the surface brightness derived from our ACS photometry.

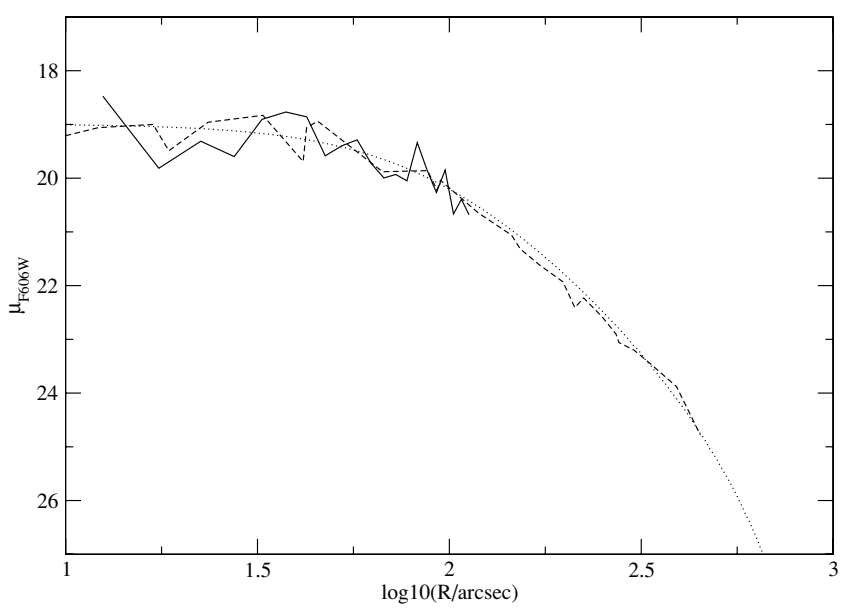

Figure 13. Surface brightness profile for NGC 6362. The dotted line is the surface brightness from our King model, the dashed line is the observation from Trager et al. (1995), and the solid line is the surface brightness derived from our ACS photometry.

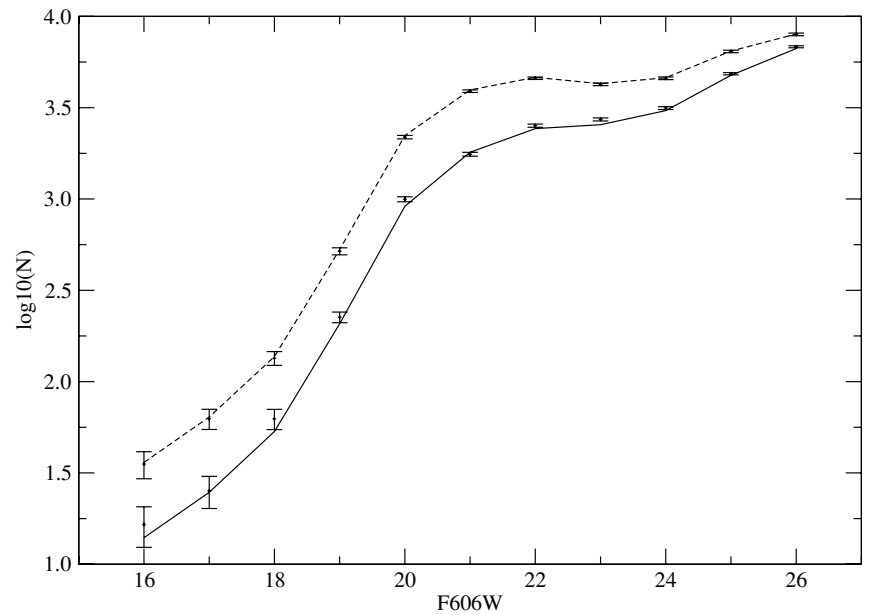

Figure 14. Local observed and model LFs for NGC 1261. The dashed line is the model LF for the 75-110 arcsec annulus, while the solid line is the model LF from the 25 to 50 arcsec annulus. The observed LFs in the two annuli are shown with error bars.

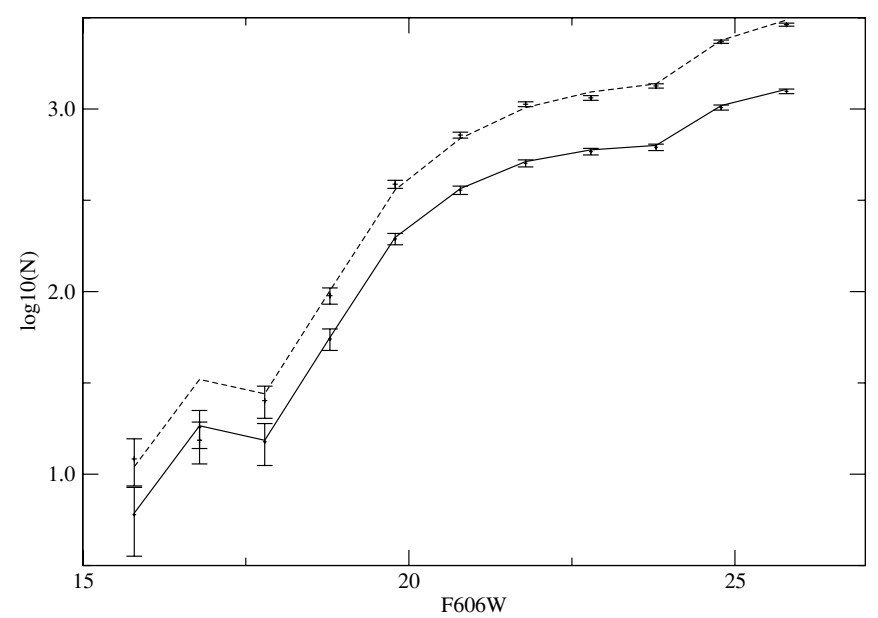

Figure 15. Local observed and model LFs for NGC 5466. The dashed line is the model LF for the 75-110 arcsec annulus, while the solid line is the model LF from the 25 to 50 arcsec annulus. The observed LFs in the two annuli are shown with error bars.

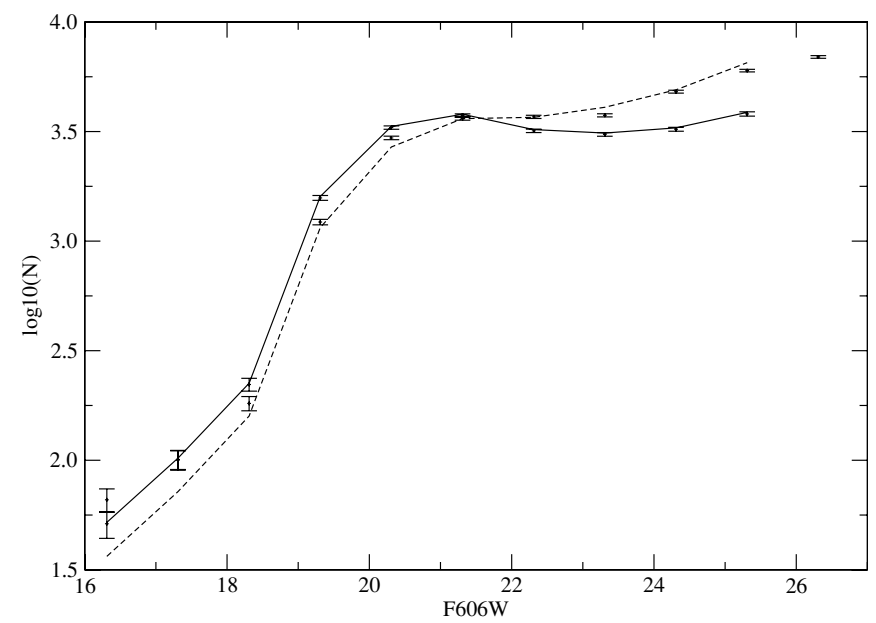

Figure 16. Local observed and model LFs for NGC 5927. The dashed line is the model LF for the 75 to 110 arcsec annulus, while the solid line is the model LF from the 25 to 50 arcsec annulus. The observed LFs in the two annuli are shown with error bars. 


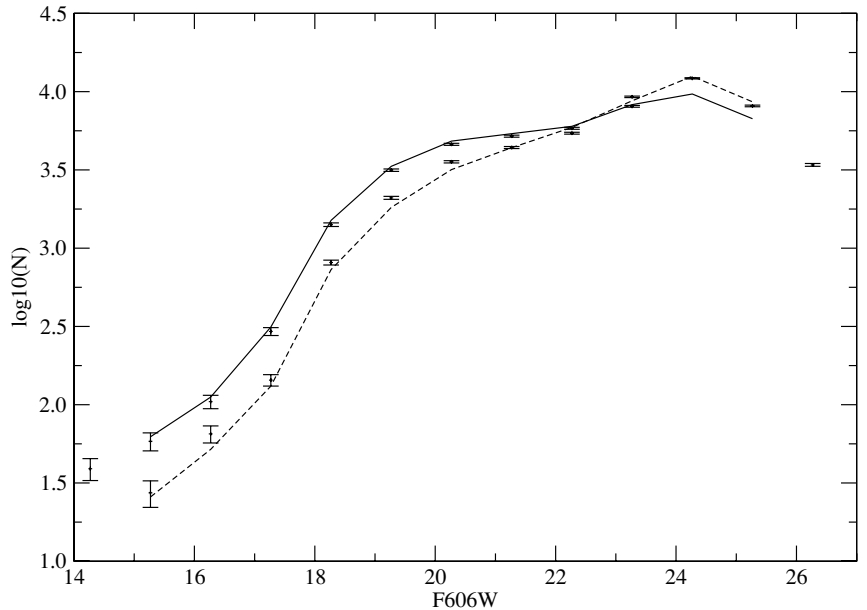

Figure 17. Local observed and model LFs for NGC 6341. The dashed line is the model LF for the 75 to 110 arcsec annulus, while the solid line is the model LF from the 25 to 50 arcsec annulus. The observed LFs in the two annuli are shown with error bars.

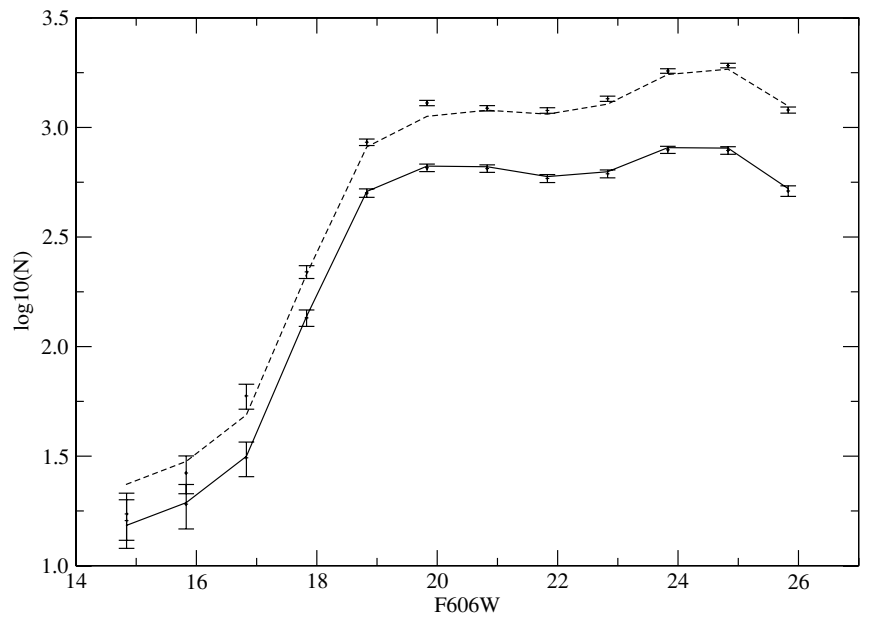

Figure 18. Local observed and model LFs for NGC 6362. The dashed line is the model LF for the 75 to 110 arcsec annulus, while the solid line is the model LF from the 25 to 50 arcsec annulus. The observed LFs in the two annuli are shown with error bars.

attempted to adjust their masses to account for unresolved companions since the mass ratio distribution is not well constrained. Our prime interest is the shape of the mass function rather than the absolute numbers; thus, unresolved binaries only influence the results if the companion-star mass function is strongly biased toward a particular mass range. Estimates of the overall binary fraction in globular clusters vary, but the color-magnitude data indicate that binarity is unlikely to exceed $20 \%$ in any clusters include in this sample. This issue is being examined in more detail by A. P. Milone et al. (2010, in preparation).

The global present-day mass functions can be seen in Figure 19. Those mass functions cover the mass range from the MSTO down to the $50 \%$ completeness limit. In most cases, the mass functions extend to $0.2 M_{\odot}$, but for the more distant clusters the lower mass limit is closer to $\sim 0.35 M_{\odot}$. As discussed further in Section 6.1, we have fit power laws to each mass function, and the derived slopes are given in Table 3 . The King models include evolved stars on the RGB, but these mass bins are not shown in Figure 19. Instead the global mass functions are truncated to start at the MSTO and continue down the MS. The slight mass difference from bin to bin and higher uncer-

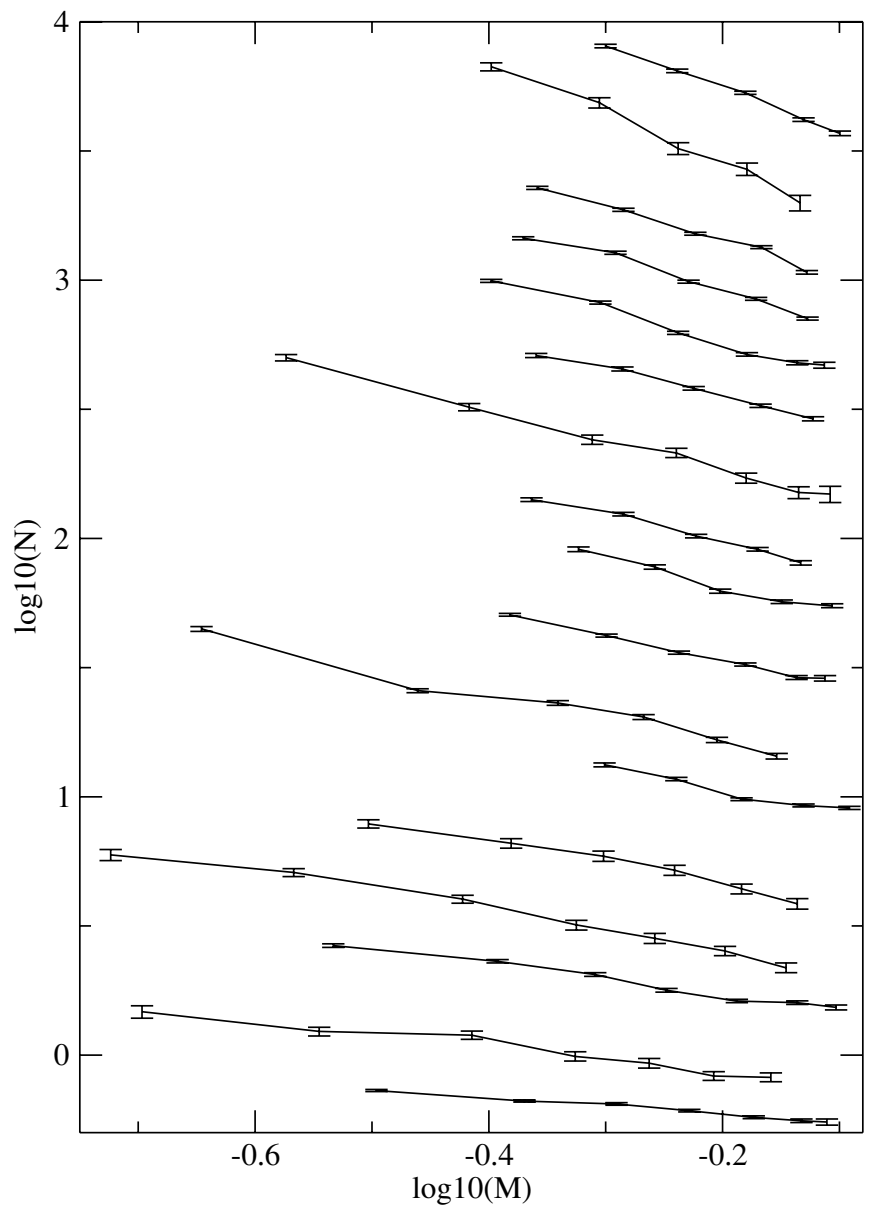

Figure 19. Global present-day mass functions for the clusters under investigation. The clusters are sorted by slope with arbitrary scaling on the $y$-axis. From top to bottom, the clusters are NGC $362(\alpha=-1.69)$, NGC $5053(\alpha=-1.46)$, NGC $6093(\alpha=-1.36)$, NGC $5272(\alpha=-1.31)$, NGC $6341(\alpha=$ $-1.23)$, NGC $5904(\alpha=-1.15)$, NGC $5466(\alpha=-1.15)$, NGC 6541 $(\alpha=-1.07)$, NGC $5927(\alpha=-1.07)$, NGC $6205(\alpha=-0.98)$, NGC 7099 $(\alpha=-0.92)$, NGC $104(\alpha=-0.84)$, NGC $288(\alpha=-0.83)$, NGC 3201 $(\alpha=-0.77)$, NGC $1261(\alpha=-0.59)$, NGC $6362(\alpha=-0.49)$, and NGC 5286 $(\alpha=-0.29)$. The power law is given by the form $N \propto M^{\alpha}$.

tainty caused by the small number of bins clutter the high-mass end of the MF and the extra points do not change the derived mass function slope.

\section{DISCUSSION}

\subsection{The Global Mass Functions}

Several clusters in the present sample have previous $H S T$ observations (Table 1). In most cases, it is not possible to compare the results, since the published data are local mass functions, uncorrected for mass segregation, and therefore tend to underestimate the proportion of low-mass stars. The exception is the analysis by Piotto \& Zoccali (1999), which employs the same formalism used in this paper to extrapolate to global mass functions. The two clusters in common are NGC 6341 (M92) and NGC 7099 (M30), and the results are in reasonable agreement. Characterizing the mass function as a power law, (Piotto \& Zoccali 1999) derive slopes of -1.1 and -1.2 , respectively, while our values are -1.2 and -0.9 . We note that these slopes are close to the values used to characterize the mass function of local Galactic disk stars in the same mass range (Reid et al. 2002). 
Figure 19 shows that most of the derived mass functions are more than adequately represented by a single power law over the full mass range covered by the present observations. There are three exceptions, NGC 5466, NGC 5927, and NGC 6093, where the present measurements deviate from the fit at the lowest masses. It is unclear whether this discrepancy is a signature of preferential tidal stripping of low-mass stars, or whether it reflects the shape of the IMF. The break in the mass function lies at a different mass in each cluster: $0.27 M_{\odot}$ in NGC 5466; $0.47 M_{\odot}$ in NGC 5927; and $0.44 M_{\odot}$ in NGC 6093. Thus, the results that we derive from our analysis do not support the hypothesis put forward by Paresce \& de Marchi (2000) that cluster mass functions are well matched by lognormal distributions, with a common characteristic mass of $M_{c} \sim$ $0.35 M_{\odot}$.

\subsection{Correlations with Other Parameters}

A prime goal of the present survey is to investigate potential correlations between the form of the present-day mass function and other intrinsic and extrinsic cluster parameters, particularly metallicity. The key question is whether the diversity of mass function slopes given in Table 3 is more closely related to intrinsic variations in the cluster IMF, or whether the variation is dominated by evolutionary effects, such as tidal stripping.

This issue has been addressed by a number of previous studies. In particular, Capaccioli et al. (1993) were the first to suggest that cluster mass functions were dependent on the location of the cluster within the Milky Way, potentially indicating that dynamical evolution plays a role in determining the form present-day mass function. Djorgovski et al. (1993) appeared to confirm this result, analyzing ground-based data for a larger sample of clusters. Their analysis suggested a mild residual dependence on metallicity, with metal-poor clusters having a steeper mass function, i.e., a higher proportion of lowmass stars. This dependence runs contrary to recent theoretical modeling (Larson 1998). We note that there are six clusters in common with the present sample (NGC 104, 5053, 5272, 5904, 6341, and 7099). In four cases, we derive significantly flatter slopes for the global mass function: thus, Djorgovski et al. (1993) assign values of $\alpha=-0.5,-2.35,-1.7$, and -2 to NGC 5053, 5272, 5904, and 6341 (note that they tabulate the power-law slopes as $x_{0}$, where a Salpeter mass function has $x_{0}=1.35$; in the convention used here, a Salpeter mass function has $\alpha=-2.35$ ); we derive power-law slopes of $-1.46,-1.31$, -1.15 , and -1.23 , respectively.

Finally, Piotto \& Zoccali (1999) used HST observations of seven clusters (M10, M15, M22, M30, M55, M92, and NGC 6397) to probe this issue. As noted above, M92 (NGC 6341) and M30 (NGC 7099) are included in our present analysis. They use a similar formalism to that adopted in the present paper to derive global mass functions, which they characterize as power laws. They find weak correlations with Galactic location, the destruction rate and the half-mass relaxation time, all suggestive of dynamical effects. They also echo Djorgovski et al. (1993) in finding a mild correlation with metallicity.

Previous investigations have had to rely on data from diverse sources and, in the case of HST-based analyses, small sample size. We have the advantage of a homogeneous data set that has been reduced and analyzed in a self-consistent fashion. Moreover, our 17 clusters matches the Djorgovski et al. (1993) sample size, and more than doubles the number of clusters with HST data available to Piotto \& Zoccali (1999). Scouring the
Harris (1996) catalog of cluster properties, we have identified over 30 different parameters associated with each cluster. Those parameters include galactic latitude and longitude, metallicity, core and tidal radii, and stellar population properties, such as the specific frequency of RR Lyrae stars. For consistency with previous analyses, we correlate against both values from Harris (1996) and results from our own analysis when there are discrepancies. We also consider the destruction timescale, as computed by Gnedin \& Ostriker (1997). Tables 2-4 assemble those parameters for the clusters in the present sample.

Some previous investigations, e.g., Djorgovski et al. (1993), have used Principal Component Analysis (PCA) to probe parametric correlation. Since we are focused on the potential source(s) of variation in the mass function, we consider pairwise correlations between $\alpha$, the slope of the power-law fit to the mass function, and other parameters.

Formally, the statistical correlation between two parameters $(X, Y)$ is given by

$$
r(X, Y)=\frac{\operatorname{cov}(X, Y)}{\sigma_{X} \sigma_{Y}}
$$

where

$$
\operatorname{cov}(X, Y)=\sum_{i=1}^{N} \frac{\left(x_{i}-\bar{x}\right)\left(y_{i}-\bar{y}\right)}{N}
$$

and

$$
\sigma=\sqrt{\frac{1}{N} \sum_{i=1}^{N}\left(x_{i}-\bar{x}\right)^{2}} .
$$

We have computed linear correlations, $r$, between the presentday mass function slope and the parameters listed in Tables 2-4. A Monte Carlo simulation with 500,000 realizations was completed to investigate how uncertainties in fitting power laws to the individual mass functions affect the correlations. The results are given in Table 5, where we also list $r^{2}$. The latter measures the proportion of the variation in one parameter that can be attributed to another parameter, i.e., $r^{2}=0.34$ suggests that $34 \%$ of the variation of one parameter is related to the other. We emphasize that correlation does not necessarily indicate causality; since we are looking at pair-wise correlations, it is entirely possible for correlations to occur through mutual dependence on a third parameter. The individual values of $r^{2}$, together with the associated uncertainties, are shown in Figure 20.

A quick scan of Table 5 shows that most parameters have extremely low correlations. These include the core and tidal radii, and the degree of central concentration. de Marchi $\&$ Pulone (2007) have suggested that there is a correlation between the last parameter, $c$, and $\alpha$. Figure 21 plots the distribution of those parameters from that analysis and from the present investigation. A number of clusters are common to both analyses; in some cases, we derive different mass function slopes; in others, the concentration parameter is different since we derived different values for $R_{t}$ and/or $R_{c}$. The separate results for the same cluster are associated in the figure. de Marchi et al. argued that the results were broadly consistent with a relation $\alpha=\frac{2.3}{c} 2.5$. Our data are at best only weakly consistent with that hypothesis, and suggest little or no correlation.

The low level of correlations between $\alpha$ and $R_{t}, R_{c}$, and $c$ indicates that the mass function is not dependent on the current structural parameters. There is also a poor correlation between the mass function slope and metallicity, with $r \sim 0.24$ 
Table 4

Cluster Parameters

\begin{tabular}{|c|c|c|c|c|c|c|c|c|c|c|c|c|}
\hline ID & $\begin{array}{l}R_{\text {Sun }} \\
(\mathrm{kpc})\end{array}$ & $\begin{array}{c}R_{\mathrm{GC}} \\
(\mathrm{kpc})\end{array}$ & $\begin{array}{c}X \\
(\mathrm{kpc})\end{array}$ & $\begin{array}{c}Y \\
(\mathrm{kpc})\end{array}$ & $\begin{array}{c}Z \\
(\mathrm{kpc})\end{array}$ & $V_{\mathrm{HB}}$ & $(m-M)_{V}$ & $V_{t}$ & $M_{V t}$ & $S_{\mathrm{RR}}$ & HBR & $\begin{array}{c}v_{r} \\
\left(\mathrm{~km} \mathrm{~s}^{-1}\right)\end{array}$ \\
\hline NGC 104 & 4.5 & 7.4 & 1.9 & -2.6 & -3.2 & 14.06 & 13.37 & 3.95 & -9.42 & 0.2 & -0.99 & -18.7 \\
\hline NGC 288 & 8.8 & 12 & -0.1 & 0 & -8.8 & 15.44 & 14.83 & 8.09 & -6.74 & 4 & 0.98 & -46.6 \\
\hline NGC 362 & 8.5 & 9.4 & 3.1 & -5 & -6.2 & 15.44 & 14.81 & 6.4 & -8.41 & 3 & -0.87 & 223.5 \\
\hline NGC 1261 & 16.4 & 18.2 & 0.1 & -10.1 & -12.9 & 16.70 & 16.10 & 8.29 & -7.81 & 13.5 & -0.71 & 68.2 \\
\hline NGC 3201 & 5 & 8.9 & 0.6 & -4.9 & 0.8 & 14.77 & 14.21 & 6.75 & -7.46 & 80.1 & 0.08 & 494 \\
\hline NGC 5053 & 16.4 & 16.9 & 2.9 & -1.3 & 16.1 & 16.65 & 16.19 & 9.47 & -6.72 & 18.4 & 0.52 & 44 \\
\hline NGC 5272 & 10.4 & 12.2 & 1.5 & 1.4 & 10.2 & 15.68 & 15.12 & 6.19 & -8.93 & 49 & 0.08 & -147.6 \\
\hline NGC 5286 & 11 & 8.4 & 7.2 & -8.1 & 2 & 16.5 & 15.95 & 7.34 & -8.61 & 4.7 & 0.8 & 57.4 \\
\hline NGC 5466 & 15.9 & 16.2 & 3.3 & 3.0 & 15.2 & 16.47 & 16.00 & 9.04 & -6.96 & 32.8 & 0.58 & 107.7 \\
\hline NGC 5904 & 7.5 & 6.2 & 5.1 & 0.3 & 5.4 & 15.07 & 14.46 & 5.65 & -8.81 & 37.7 & 0.31 & 52.6 \\
\hline NGC 5927 & 7.6 & 4.5 & 6.3 & -4.2 & 0.6 & 16.55 & 15.81 & 8.01 & -7.80 & 0.0 & -1.00 & -107.5 \\
\hline NGC 6093 & 10.0 & 3.8 & 9.4 & -1.2 & 3.3 & 16.10 & 15.56 & 7.33 & -8.23 & 3.1 & 0.93 & 8.2 \\
\hline NGC 6205 & 6.8 & 8.2 & 2.6 & 4.4 & 4.4 & 14.90 & 14.21 & 5.78 & -8.43 & 2.1 & 0.97 & -246.6 \\
\hline NGC 6341 & 8.2 & 9.6 & 2.5 & 6.3 & 4.7 & 15.10 & 14.64 & 6.44 & -8.20 & 13.1 & 0.91 & -120.3 \\
\hline NGC 6362 & 7.6 & 5.1 & 5.9 & -4.1 & -2.3 & 15.33 & 14.67 & 7.73 & -6.94 & 55.1 & -0.58 & -13.1 \\
\hline NGC 6541 & 7 & 2.2 & 6.8 & -1.3 & -1.4 & 15.2 & 14.67 & 6.3 & -8.37 & 0 & 1 & -158.7 \\
\hline NGC 7099 & 8.0 & 7.1 & 4.9 & 2.5 & -5.9 & 15.93 & 14.62 & 7.19 & -7.43 & 3.2 & 0.89 & -181.9 \\
\hline ID & $\begin{array}{c}v_{\mathrm{LSR}} \\
\left(\mathrm{km} \mathrm{s}^{-1}\right)\end{array}$ & $c$ & $\begin{array}{c}e \\
\left(\mathrm{~km} \mathrm{~s}^{-1}\right)\end{array}$ & $\begin{array}{c}R_{c} \\
(\operatorname{arcmin})\end{array}$ & $\begin{array}{c}R_{h} \\
(\operatorname{arcmin})\end{array}$ & $\begin{array}{c}R_{t} \\
(\operatorname{arcmin})\end{array}$ & $\begin{array}{c}\log (t c) \\
(\mathrm{yr})\end{array}$ & $\begin{array}{c}\log (t h) \\
(\mathrm{yr})\end{array}$ & 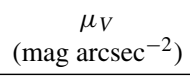 & $\begin{array}{c}\rho_{0} \\
\left(L_{\odot} \mathrm{pc}^{-3}\right)\end{array}$ & Destruction Rate & \\
\hline NGC 104 & -28 & 2.03 & 0.09 & 0.4 & 2.79 & 42.86 & 8.74 & 9.2 & 17.65 & 2.96 & 0.08 & \\
\hline NGC 288 & -53.9 & 0.96 & 0.0 & 1.42 & 2.22 & 12.94 & 8.33 & 8.19 & 23.1 & 1.11 & 19.3 & \\
\hline NGC 362 & 213.3 & 1.94 & 0.01 & 0.19 & 0.81 & 16.11 & 7.49 & 8.67 & 17.63 & 3.48 & 0.23 & \\
\hline NGC 1261 & 53.4 & 1.27 & 0.07 & 0.39 & 0.75 & 7.28 & 7.34 & 8.02 & 20.93 & 2.22 & 0.25 & \\
\hline NGC 3201 & 481.9 & 1.3 & 0.12 & 1.43 & 2.68 & 28.45 & 8.78 & 9.2 & 18.77 & 2.73 & 0.27 & \\
\hline NGC 5053 & 51.8 & 0.84 & 0.21 & 1.98 & 3.5 & 13.67 & 9.05 & 10 & 16.77 & 3.37 & 13.9 & \\
\hline NGC 5272 & -137 & 1.84 & 0.04 & 0.55 & 1.12 & 38.19 & 8.41 & 9.02 & 16.07 & 4.17 & 0.04 & \\
\hline NGC 5286 & 54.7 & 1.46 & 0.12 & 0.29 & 0.69 & 8.36 & 9.56 & 9.75 & 21.28 & 0.88 & 0.4 & \\
\hline NGC 5466 & 119.7 & 1.32 & 0.11 & 1.64 & 2.25 & 34.24 & 8.26 & 9.53 & 16.05 & 3.91 & 3.01 & \\
\hline NGC 5904 & 65.7 & 1.83 & 0.14 & 0.42 & 2.11 & 28.4 & 8.29 & 8.98 & 17.45 & 3.87 & 0.11 & \\
\hline NGC 5927 & -106.3 & 1.60 & 0.04 & 0.42 & 1.15 & 16.68 & 8.94 & 9.23 & 17.56 & 3.32 & 0.24 & \\
\hline NGC 6093 & 18.6 & 1.95 & 0.00 & 0.15 & 0.65 & 13.28 & 7.73 & 8.86 & 15.19 & 4.76 & 4.20 & \\
\hline NGC 6205 & -228.2 & 1.49 & 0.9 & 0.88 & 1.49 & 28.3 & 8.94 & 8.96 & 16.80 & 3.33 & 3.17 & \\
\hline NGC 6341 & -101.7 & 1.81 & 0.10 & 0.23 & 1.09 & 15.17 & 7.84 & 9.06 & 15.58 & 4.29 & 0.27 & \\
\hline NGC 6362 & -15.1 & 1.10 & 0.07 & 1.32 & 2.18 & 16.67 & 8.57 & 8.69 & 21.24 & 2.42 & 0.48 & \\
\hline NGC 6541 & -152.7 & 2 & 0.12 & 0.3 & 1.19 & 29.6 & 5.09 & 8.4 & 17.13 & 5.73 & 0.24 & \\
\hline NGC 7099 & -176.3 & 2.50 & 0.06 & 0.06 & 1.15 & 18.34 & 8.93 & 9.20 & 18.90 & 2.35 & 0.29 & \\
\hline
\end{tabular}

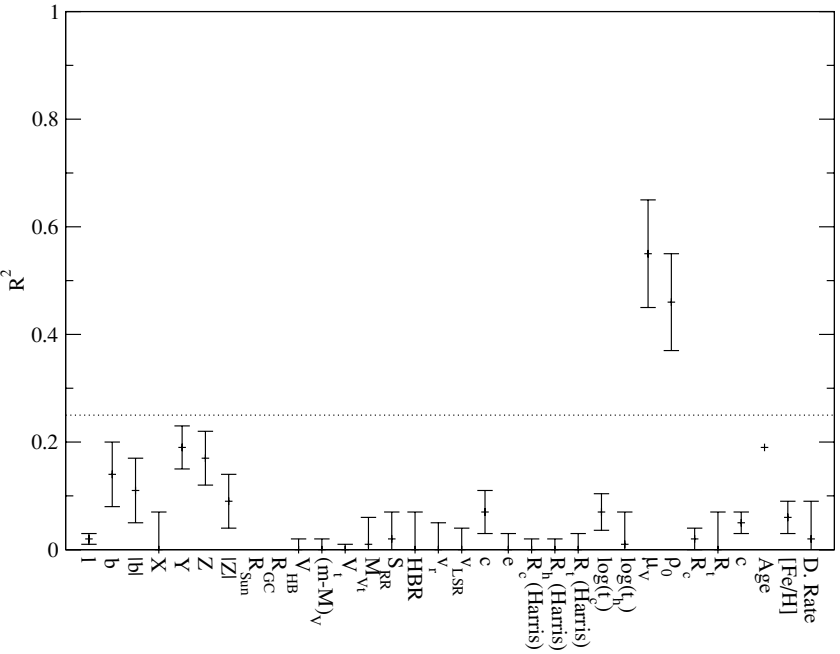

Figure 20. Correlation coefficient squared between the mass function slope and a variety of other cluster parameters. Parameters above the dotted line have effects on the present-day mass function slope greater than $25 \%$.

or $r^{2} \sim 6 \%$. This result holds whether we use the best-fit isochrone metallicities, or literature values (see Figure 22).
Thus, the present sample does not confirm the mild correlation with $[\mathrm{Fe} / \mathrm{H}]$ hypothesized by Djorgovski et al. (1993) and Piotto \& Zoccali (1999).

Similarly, we do not confirm the strong correlation between Galactic location and mass function slope postulated by Capaccioli et al. (1993), Djorgovski et al. (1993), and Piotto \& Zoccali (1999). We find no correlation with $R_{\mathrm{GC}}$, as hypothesized by Djorgovski et al. (1993). The correlation coefficients measured for $|b|$ and $|Z|$ (where $Z$ is height above the Plane, not metallicity) are -0.33 and -0.30 , respectively (Figure 22 ); those parameters therefore can only account for less than $10 \%$ of the dispersion in mass function slope. Indeed, there are stronger correlation between $\alpha$ and the Galactic coordinates $Y$ and $Z$, with latitudes, $b$, and with the isochrone estimates, than with the absolute values of latitude or distance from the plane.

The strongest correlations evident in this analysis, and the only correlations of strong statistical significance, are between mass function slope and the central surface brightness, $\mu_{V}$, and the central density, $\rho_{0}$ (Figure 22). These two parameters are directly related, since $\rho_{0}=\frac{\mu_{V}}{\left(r_{c} p\right)}$, where $r_{c}$ the core radius and $p$ is a concentration index. Thus, both parameters are essentially measuring the same physical quantity, the central concentration of the cluster. The correlation is such that clusters 


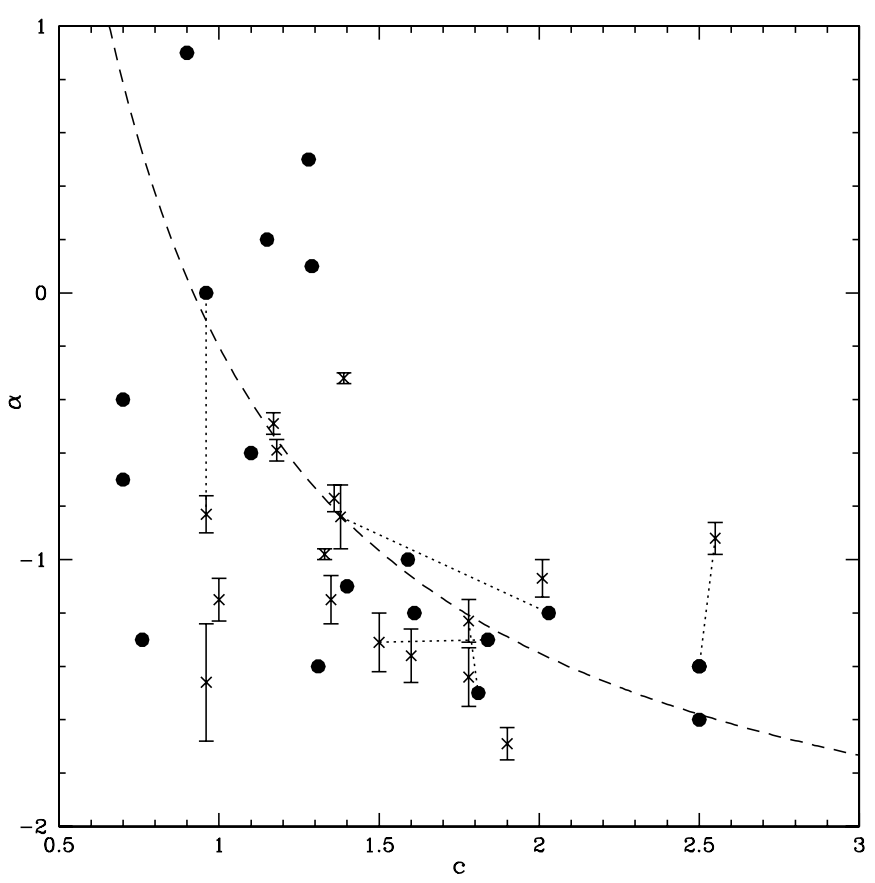

Figure 21. Relation between the slope of the best-fit power-law mass function and the degree of concentration, $c$. Crosses mark data from the present analysis, and solid points plot data from de Marchi \& Pulone (2007). A number of clusters are included in both samples, and in most cases there are differences in either $\alpha$ or the derived concentration parameter; the dotted lines connect separate measurements of the same cluster. The dashed line shows the mean relation proposed by de Marchi et al., $\alpha=\frac{2.3}{c} 2.5$.

Table 5

Parameter Correlations with PDMF Slope

\begin{tabular}{|c|c|c|}
\hline Parameter & Correlation & Correlation $^{2}$ \\
\hline$l$ & $0.14 \pm 0.08$ & $0.02 \pm 0.01$ \\
\hline$b$ & $-0.37 \pm 0.05$ & $0.14 \pm 0.06$ \\
\hline$|b|$ & $-0.33 \pm 0.05$ & $0.11 \pm 0.06$ \\
\hline$X$ & $-0.02 \pm 0.08$ & $0.00 \pm 0.07$ \\
\hline$Y$ & $-0.44 \pm 0.07$ & $0.19 \pm 0.04$ \\
\hline$Z$ & $-0.41 \pm 0.04$ & $0.17 \pm 0.05$ \\
\hline$|Z|$ & $-0.30 \pm 0.04$ & $0.09 \pm 0.05$ \\
\hline$R_{\text {Sun }}$ & $-0.11 \pm 0.05$ & $-0.11 \pm 0.02$ \\
\hline$R_{\mathrm{GC}}$ & $-0.06 \pm 0.06$ & $-0.06 \pm 0.05$ \\
\hline$V_{\mathrm{HB}}$ & $-0.03 \pm 0.07$ & $0.00 \pm 0.02$ \\
\hline$(m-M)_{V}$ & $-0.05 \pm 0.06$ & $0.00 \pm 0.02$ \\
\hline$V_{t}$ & $0.03 \pm 0.07$ & $0.00 \pm 0.01$ \\
\hline$M_{V t}$ & $0.09 \pm 0.07$ & $0.01 \pm 0.05$ \\
\hline$S_{\mathrm{RR}}$ & $0.14 \pm 0.04$ & $0.02 \pm 0.05$ \\
\hline HBR & $-0.04 \pm 0.09$ & $0.00 \pm 0.07$ \\
\hline$v_{r}$ & $0.04 \pm 0.08$ & $0.00 \pm 0.05$ \\
\hline$v_{\mathrm{LSR}}$ & $0.01 \pm 0.09$ & $0.00 \pm 0.04$ \\
\hline$c$ & $-0.27 \pm 0.07$ & $0.07 \pm 0.04$ \\
\hline$e$ & $0.07 \pm 0.07$ & $0.00 \pm 0.03$ \\
\hline$R_{\mathrm{c}}$ (Harris) & $0.04 \pm 0.05$ & $0.00 \pm 0.02$ \\
\hline$R_{\mathrm{h}}$ (Harris) & $0.00 \pm 0.06$ & $0.00 \pm 0.02$ \\
\hline$R_{\mathrm{t}}$ (Harris) & $-0.18 \pm 0.05$ & $0.00 \pm 0.03$ \\
\hline $\log (t c)$ & $-0.27 \pm 0.08$ & $0.07 \pm 0.034$ \\
\hline $\log (t h)$ & $-0.11 \pm 0.05$ & $0.01 \pm 0.06$ \\
\hline$\mu_{V}$ & $0.74 \pm 0.08$ & $0.55 \pm 0.10$ \\
\hline$\rho_{0}$ & $-0.67 \pm 0.09$ & $0.46 \pm 0.09$ \\
\hline$R_{\mathrm{c}}$ & $0.14 \pm 0.06$ & $0.02 \pm 0.02$ \\
\hline$R_{\mathrm{t}}$ & $0.00 \pm 0.09$ & $0.00 \pm 0.07$ \\
\hline$c$ & $-0.22 \pm 0.064$ & $0.05 \pm 0.02$ \\
\hline Age & $0.43 \pm 0.053$ & $0.19 \pm 0.00$ \\
\hline$[\mathrm{Fe} / \mathrm{H}]$ & $0.24 \pm 0.09$ & $0.06 \pm 0.03$ \\
\hline Destruction rate & $-0.13 \pm 0.09$ & $0.02 \pm 0.07$ \\
\hline
\end{tabular}
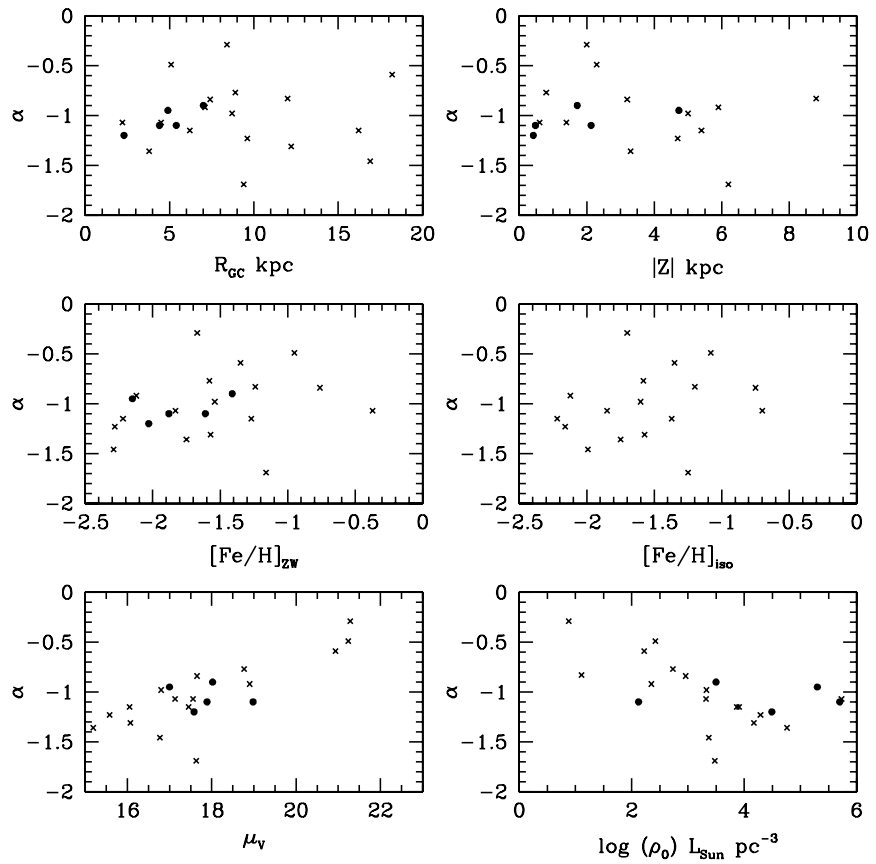

Figure 22. Correlations between the mass function slope and the following cluster parameters: Galactocentric distance $\left(R_{\mathrm{GC}}\right)$, distance above the Galactic plane $(|Z|)$, the Zinn-West metallicity $([\mathrm{Fe} / \mathrm{H}])$, the metallicity derived from the isochrone fits in the present paper, the central surface brightness $\left(\mu_{V}\right)$, and the central stellar density $\left(\rho_{0}\right)$. Crosses mark data from the present analysis; solid points plot data for five additional clusters with HST data that were analyzed by Piotto \& Zoccali (1999). The only statistically significant correlations are with $\mu_{V}$ and $\rho_{0}$.

with high central surface brightness (dense cores) tend to have steeper mass functions. We do not, however, find a significant correlation between $\alpha$ and the relaxation time at the half-mass

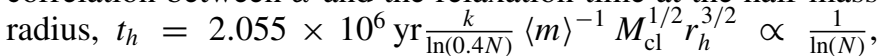
where $N$ is the total number of stars, $r_{h}$ is the half-mass radius, and $M_{\mathrm{cl}}$ is the total mass of the cluster (Binney \& Tremaine 1988).

\section{DISCUSSION}

Interpreting correlations between observational parameters always requires a degree of conjecture. In particular, one must be careful to avoid the pitfalls of assuming that post hoc necessarily implies propter hoc. Nonetheless, the correlation between central density and the slope of the stellar mass function suggests that dynamical evolution and tidal stripping may play a significant role in determining the present-day mass function. Clusters with dense cores have high binding energy, and are therefore less susceptible to depletion of low-mass stars through gravitational interactions. The absence of a correlation with $t_{h}$ suggests that the density profile is more important than the total mass.

The possibility that dynamical evolution plays a strong role in determining the present-day mass function in globular clusters has been raised by several previous studies, notably those by Capaccioli et al. (1993), Djorgovski et al. (1993), and Piotto \& Zoccali (1999) discussed in the previous section. However, those conclusions were based largely on an inferred dependence on mass function slope with location in the Galaxy: clusters at large distances from the Galactic Plane and/or Galactic center appeared to have, on average, steeper mass functions; this correlation was attributed to less frequent and weaker 
gravitational interactions with the disk and bulge. We do not confirm that dependence, although there is a suggestion of a mild correlation between $\alpha$ and $R_{\mathrm{GC}}$ for Galactocentric distances less than $8 \mathrm{kpc}$, and we note that van den Bergh et al. (1991) found evidence for a correlation between cluster diameter and Galactic centric radius. Confirmation of those putative correlations will require a larger sample. There is relatively little overlap between the current sample and the clusters considered in previous analyses. Thus, we must consider whether differences in sample selection might be responsible for the different conclusions.

Piotto \& Zoccali (1999) analyzed HST data using techniques very similar to those employed in the current paper, and there is reasonable agreement $(\delta \alpha<0.2)$ with our results for the two clusters in common. Hence, we have combined their results for the other five clusters (M10, M15, M22, M55, and NGC 6297) with our data set and repeated the analysis. The individual data points are plotted separately in Figure 22, and there was no significant impact on the derived correlations. In particular, the $r^{2}$ values drop to 0.08 for $|b|, 0.07$ for $|Z|$, and 0.00 for $R_{\mathrm{GC}}$, but remain high at 0.52 and 0.47 for $\mu_{V}$ and $\rho_{0}$, respectively.

The Djorgovski et al. (1993) sample includes 11 clusters that are not in the present sample; thus, there is more scope for discrepancies to arise in sampling the underlying distribution of parameters such as Galactic location and metallicity. However, inspection shows no obvious systematic differences in this respect. In particular, both samples cover a comparable range in $[\mathrm{Fe} / \mathrm{H}]$ and $R_{\mathrm{GC}}$; indeed, the present sample provides better sampling at small radii, with four clusters at $R_{\mathrm{GC}}<7 \mathrm{kpc}$ as opposed to two in Djorgovski et al. (1993). More significantly, the mass function slopes derived in the latter paper span a much wider range than in the present analysis. NGC 362 is the only cluster in our sample that has a mass function slope steeper then -1.5 , with $\alpha=-1.69$. In contrast, nine clusters from Djorgovski et al. (1993) have $\alpha<-1.5$, including five with near-Salpeter slopes of $\alpha<-2$. As noted in the previous section, we derive significantly flatter mass functions for four of those clusters. The Djorgovski et al. (1993) analysis is based on ground-based data and employed approximate corrections for mass segregation. These differences likely account for the steeper mass functions derived in that analysis, and the consequent apparent correlations identified in that earlier analysis.

The results presented in Table 5 lead to an interesting conclusion. If dynamical evolution is responsible for the correlation between $\mu_{V}$ and $\alpha$, then the absence of a significant correlation between $\alpha$ and Galactic location implies that these clusters have experienced similar range of gravitational interactions, regardless of their particular Galactic orbits.

The correlation coefficients listed in Table 5 indicate that $\sim 50 \%$ of the dispersion in $\alpha$ can be associated with the variation in central concentration. We have fitted a linear relation to the $\mu_{V}-\alpha$ relation, and correlated the residuals against the other cluster parameters listed in Table 5 to search for secondary correlations. Using $R^{2}>0.25$ as the definition of statistically significant, we find no significant correlations. Despite the lack of clear answers, some hints did emerge that the destruction rate from Gnedin \& Ostriker (1997) and the total cluster luminosity may be correlated. Confirmation of these correlations will require a larger sample of clusters.

\section{CONCLUSIONS}

Using observations obtained in the course of the ACS Globular Cluster Survey, we have derived luminosity functions for 17 globular clusters, spanning a wide range of metallicity and
Galactic location. Matching the observed CMDs against theoretical models, we have estimated distances and metallicities, and used the appropriate mass-luminosity relations to derive local, present-day mass functions. These data are statistically complete to $\sim 0.2-0.3 M_{\odot}$. We have used multi-mass King models to correct for mass segregation, and have extrapolated the observations to determine global mass function for each cluster. The resultant data are well matched by power laws.

We have searched for linear correlations between the slope, $\alpha$, of the power-law fit to the mass function and a wide range of other parameters. The only significant correlations that we find are with the central surface brightness and the derived central density. Unlike previous analyses, we do not find significant correlations with either Galactic location or metallicity.

The sense of the observed correlation is that clusters with high central densities tend to have steeper mass functions. Relaxation processes within each cluster lead to mass segregation, with lower mass stars subject to escape through evaporation and tidal stripping. Thus, the observed correlation is consistent with the hypothesis that the higher gravitational binding energy in the more concentrated clusters has minimized those dynamical effects.

The steepest power-law mass functions derived for clusters in the present sample of clusters have $\alpha \sim-1.4$, which is close to the power-law slope derived in investigations of the mass function of local disk dwarfs (Reid et al. 2002; Kroupa 2002). This opens the possibility that there is a universal IMF and the observed variation among globular cluster systems is related to evolutionary effects over the life of the cluster. We are currently extending our analysis to include the remaining clusters in the ACS GGC, and the addition of those systems will provide further insight into this issue.

We thank Ivan King for providing critical assessment and insightful comments regarding the analysis methods and results presented in this paper. Support for US investigators in program GO-10775 was provided by NASA through a grant from the Space Telescope Science Institute, which is operated by the Association of Universities for Research in Astronomy, Inc., under NASA contract NAS 5-26555. A.A., A.M., and A.R. are funded by the Instituto de Astrofisica de Canarias (grant 310394) and by the Ministry of Education and Science of the Kingdom of Spain (grant AYA-2008-67913).

\section{REFERENCES}

Albrow, M. D., de Marchi, G., \& Sahu, K. C. 2002, ApJ, 579, 660

Andersen, M., Meyer, M. R., Greissl, J., \& Aversa, A. 2008, ApJ, 683, L183

Anderson, J. 1997, PhD thesis, Univ. of California, Berkeley

Anderson, J., \& King, I. R. 2006, ACS Instrument Science Report, ACS ISR 2006-01

Anderson, J., et al. 2008, AJ, 135, 2055

Andreuzzi, G., Buonanno, R., Fusi Pecci, F., Iannicola, G., \& Marconi, G. 2000, A\&A, 353, 944

Bahcall, J. N., \& Schneider, D. P. 1988, in IAU Symp. 126, The Harlow-Shapley Symposium on Globular Cluster Systems in Galaxies, ed. J. E. Grindlay \& A. G. D. Philip (Dordrecht: Kluwer), 455

Bedin, L. R., Anderson, J., King, I. R., \& Piotto, G. 2001, ApJ, 560, 75

Bedin, L. R., Piotto, G., Anderson, J., Cassisi, S., King, I. R., Momany, Y., \& Carraro, G. 2004, ApJ, 605, L125

Bellazzini, M., Fusi Pecci, F., Montegriffo, P., Messineo, M., Monaco, L., \& Rood, R. T. 2002, AJ, 123, 2541

Binney, J., \& Tremaine, S. 1988, Galactic Dynamics (Princeton: Princeton Univ. Press)

Bonatto, C., \& Bica, E. 2008, A\&A, 477, 829

Bromm, V., Coppi, P. S., \& Larson, R. B. 2002, ApJ, 564, 23

Bromm, V., \& Loeb, A. 2003, Nature, 425, 812 
Burgarella, D., et al. 1994, A\&A, 287, 769

Capaccioli, M., Piotto, G., \& Stiavelli, M. 1993, MNRAS, 261, 819

Carretta, E., \& Gratton, R. G. 1997, A\&AS, 121, 95

Carretta, E., Gratton, R. G., Clementini, G., \& Fusi Pecci, F. 2000, ApJ, 533, 215

Chabrier, G. 2003, PASP, 115, 763

Cool, A. M., Piotto, G., \& King, I. R. 1996, ApJ, 468, 655

Covey, K. R., et al. 2008, AJ, 136, 1778

Cruz, K. L., et al. 2007, AJ, 133, 439

Da Costa, G. S. 1982, AJ, 87, 990

De Angeli, F., Piotto, G., Cassisi, S., Busso, G., Recio-Blanco, A., Salaris, M., Aparicio, A., \& Rosenberg, A. 2005, AJ, 130, 116

de Marchi, G. 1999, AJ, 117, 303

de Marchi, G., \& Paresce, F. 1994, ApJ, 422, 597

de Marchi, G., \& Paresce, F. 1995a, A\&A, 304, 202

de Marchi, G., \& Paresce, F. 1995b, A\&A, 304, 211

de Marchi, G., \& Paresce, F. 1997, ApJ, 476, L19

de Marchi, G., Paresce, F., \& Pulone, L. 2000, ApJ, 530, 342

de Marchi, G., \& Pulone, L. 2007, A\&A, 467, 107

Djorgovski, S., Piotto, G., \& Capaccioli, M. 1993, AJ, 105, 2148

Dotter, A., Chaboyer, B., Jevremović, D., Baron, E., Ferguson, J. W., Sarajedini, A., \& Anderson, J. 2007, AJ, 134, 376

Elson, R. A. W., Gilmore, G. F., Santiago, B. X., \& Casertano, S. 1995, AJ, 110 682

Fahlman, G. G., Richer, H. B., Searle, L., \& Thompson, I. B. 1989, ApJ, 343 , L49

Ferraro, F. R., Carretta, E., Bragaglia, A., Renzini, A., \& Ortolani, S. 1997, MNRAS, 286, 1012

Gnedin, O. Y., \& Ostriker, J. P. 1997, ApJ, 487, 667

Gratton, R. G., Bragaglia, A., Carretta, E., Clementini, G., Desidera, S., Gundahl, F., \& Lucatello, S. 2003, A\&A, 408, 529

Gratton, R., Sneden, C., \& Carretta, E. 2004, ARA\&A, 42, 385

Grillmair, C. J., \& Smith, G. H. 2001, AJ, 122, 3231

Guhathakurta, P., Yanny, B., Bahcall, J. N., \& Schneider, D. P. 1994, AJ, 108 , 1786

Guhathakurta, P., Yanny, B., Schneider, D. P., \& Bahcall, J. N. 1992, AJ, 104, 1790

Gunn, J. E., \& Griffin, R. F. 1979, AJ, 84, 752

Harris, W. E. 1993, AJ, 106, 604

Harris, W. E. 1996, AJ, 112, 1487

Holtzman, J. A., Burrows, C. J., Casertano, S., Hester, J. J., Trauger, J. T., Watson, A. M., \& Worthey, G. 1995, PASP, 107, 1065

King, I. 1958, AJ, 63, 465

King, I. R., Anderson, J., Cool, A. M., \& Piotto, G. 1998, ApJ, 492, L37

King, I. R., Sosin, C., \& Cool, A. M. 1995, ApJ, 452, L33

Kroupa, P. 2002, Science, 295, 82

Larson, R. B. 1998, MNRAS, 301, 569
Marconi, G., et al. 1998, MNRAS, 293, 479

Milone, A. P., et al. 2008, ApJ, 673, 241

Montalban, J., D’Antona, F., \& Mazzitelli, I. 2000, A\&A, 360, 935

Oort, J. H., \& van Herk, G. 1959, Bull. Astron. Inst. Netherlands, 14, 299

Paresce, F., \& de Marchi, G. 2000, ApJ, 534, 870

Paresce, F., de Marchi, G., \& Jedrewski, R. 1995a, ApJ, 442, 57

Paresce, F., de Marchi, G., \& Romaniello, M. 1995b, ApJ, 440, 216

Pasquali, A., Brigas, M. S., \& de Marchi, G. 2000, A\&A, 362, 557

Pasquali, A., de Marchi, G., Pulone, L., \& Brigas, M. S. 2004, A\&A, 428, 469

Paust, N. E. Q., Chaboyer, B., \& Sarajedini, A. 2007, AJ, 133, 278

Paust, N. E. Q., et al. 2009, AJ, 137, 246

Pietrinferni, A., Cassisi, S., Salaris, M., \& Castelli, F. 2006, ApJ, 642, 797

Piotto, G., Cool, A. M., \& King, I. R. 1997, AJ, 113, 1345

Piotto, G., King, I. R., Capaccioli, M., Ortolani, S., \& Djorgovski, S. 1990, ApJ, 350,662

Piotto, G., \& Zoccali, M. 1999, A\&A, 345, 485

Piotto, G., Zoccali, M., King, I. R., Djorgovski, S. G., Sosin, C., Dorman, B., Rich, R. M., \& Meylan, G. 1999, AJ, 117, 264

Piotto, G., et al. 2007, ApJ, 661, L53

Pollard, D. L., Sandquist, E. L., Hargis, J. R., \& Bolte, M. 2005, ApJ, 628, 729

Pulone, L., de Marchi, G., Covino, S., \& Paresce, F. 2003, A\&A, 399, 121

Pulone, L., de Marchi, G., \& Paresce, F. 1999, A\&A, 342, 440

Reid, I. N., \& Gizis, J. E. 1997, AJ, 113, 2246

Reid, I. N., Gizis, J. E., \& Hawley, S. L. 2002, AJ, 124, 2721

Richer, H. B., \& Fahlman, G. G. 1988, ApJ, 325, 218

Richer, H. B., Fahlman, G. G., Buonanno, R., Fusi Pecci, F., Searle, L., \& Thompson, I. B. 1991, ApJ, 381, 147

Richer, H. B., Fahlman, G. G., \& Vandenberg, D. A. 1988, ApJ, 329, 187

Richer, H. B., et al. 2002, ApJ, 574, L151

Richer, H. B., et al. 2008, AJ, 135, 2141

Rutledge, G. A., Hesser, J. E., \& Stetson, P. B. 1997, PASP, 109, 907

Salaris, M., Chieffi, A., \& Straniero, O. 1993, ApJ, 414, 580

Sandage, A. R. 1954, AJ, 59, 162

Santiago, B. X., Elson, R. A. W., \& Gilmore, G. F. 1996, MNRAS, 281, 1363

Sarajedini, A., et al. 2007, AJ, 133, 1658

Siegel, M. H., et al. 2007, ApJ, 667, L57

Silvestri, F., Ventura, P., D’Antona, F., \& Mazzitelli, I. 1998, ApJ, 509, 192

Sirianni, M., et al. 2005, PASP, 117, 1049

Sollima, A., Cacciari, C., \& Valenti, E. 2006, MNRAS, 372, 1675

Sollima, A., Ferraro, F. R., \& Bellazzini, M. 2007, MNRAS, 381, 1575

Sosin, C., \& Cool, A. M. 1995, ApJ, 452, L29

Tayler, R. J. 1954, AJ, 59, 413

Trager, S. C., King, I. R., \& Djorgovski, S. 1995, AJ, 109, 218

van den Bergh, S., Morbey, C., \& Pazder, J. 1991, ApJ, 375, 594

Villanova, S., et al. 2007, ApJ, 633, 296

Zinn, R., \& West, M. J. 1984, ApJS, 55, 45 